\title{
Edubba’a und Edubba’a-Literatur: Rätsel und Lösungen ${ }^{1}$
}

\author{
von Konrad Volk, Tübingen
}

\author{
Dietz Otto Edzard \\ zum 30. Juli 1999 - dem Abschied \\ vom bára-é-dub-ba-a
}

In der Zeit des Königs Samsuiluna von Babylon (1749-1712), vermutlich aber auch schon früher, kursierte im Schulbetrieb Babyloniens ein berühmtes Rätsel. Dieses Rätsel führt nicht nur auf direktem Wege zum Orte des Geschehens, sondern zeigt auch stellvertretend auf, wie viele Rätsel die schriftlichen Hinterlassenschaften Mesopotamiens uns immer wieder aufgeben, und wie problematisch manch sicher geglaubtes Allgemeingut bei genauerer Betrachtung sein kann. FolgendermaBen lautet das Rätsel ${ }^{2}$ :

„Ein Haus, wie der Himmel (auf) einem Fundament fest gegründet ${ }^{3}$.

Ein Haus - jemand hat es wie eine Schatzkiste mit einem Leintuch bedeckt.

Ein Haus, wie eine Ente auf festem Sockel stehend 4

Geschlossenen Auges trat einer ein,

geöffneten Auges kam er (wieder) heraus.

Des (Rätse]s) Lösung: Das Edubbasa".

1 Diese Untersuchung wurde durch dic finanzielle Unterstützung der Deutschen Forschungsgemeinschaft im Rahmen des Forschungsprojektes „Kindheit, Erziehung und Ausbildung in Babylonien und Assyrien" ermöglicht. Für Hinweise und kritische Anmerkungen danke ich sehr herzlich D. O. Edzard. München, und C. Wilcke. Leipzig. Für manch weiteren Hinweis bin ich zu Dank verpflichtet meinen Leipziger Kollegen J. Hazenbos. M. Müller und W. Sallaberger; H. J. Abs, Seminar für Philosophie und Erziehungswissenschaft, sowic M. A. Brandes, Orientalisches Seminar. Freiburg. M. Civil, Chicago, danke ich ganz besonders für die Möglichkeit der Einsichtnahme in sein noch unveröffentlichtes Edubba'a-Material.

2 Vgl. zuletzt M. Civil, AulOr. 5 (1987) 17 凡.

${ }^{3}$ Die beiden zur Zeile erhaltenen Textzeugen (A: UET, 6,340:1 und B: LET 6,341:1)

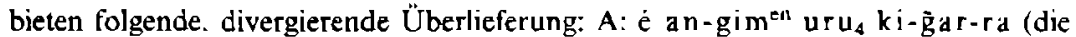
o. a. Übersetzung folgt dieser Überlieferung); B: é an-gim uru $u_{4}$ gar-ra ..Ein Haus, wie der Himmel (auf) ein Fundament fest gesetzt“.

4 Vgl. zu dieser Aussage den Edubba'd-Brief VS 17,44; Ni. 972 (ISET 1,126); BE 31,29; UM 29-13-520 (Or. 58, 1989) Z. 21 [Nibruk]-ging-nam ki-gal-la ga-bi-ib-gub „(Das Edubba'a von Isin) will ich wie (dasjenige von) Nippur auf ein Podest stellen“ (vgl. J. van Dijk, Or. 58, 451 f.). Das Bild einer auf einem Podest stehenden Ente 
Beschreiben die ersten drei Zeilen das „Haus“, das - metaphorisch auf ewig währenden Fundamenten ruht, dessen Inneres wie ein Schatz bewahrt, gehütet und dem Anblick der Unwürdigen verborgen bleibt, so widmen sich die folgenden zwei Zeilen der Wandlung dessen, der dies „Haus" betritt und schließlich wieder verläßt: Betraut mit den Schätzen des Hauses kann er als Mensch gelten, dem - aus der Perspektive der Urheber des Rätsels - die Augen für das Leben und die ihn umgebende Welt geöffnet wurden. Die letzte, einzeln stehende Zeile des Rätsels hebt schließlich, um in den Worten des Rätsels zu sprechen, das Leintuch des Geheimnisses: Es ist einzig das Edubba'a, das einem Menschen die Augen öffnen kann. Eine ebenso monopolistische wie elitäre Vorstellung ${ }^{5}$.

Folgt man der Mehrzahl der bisherigen Bearbciter' dieses Rätsels, so wäre des Rätsels Lösung und Schlüsselbegriff é-dub-ba-a ganz einfach mit ,Academy“, ,Schule“ oder, entsprechend der traditionellen babylonischen Standardübersetzung, mit bït tuppi $(m)$,Tafelhaus" wiederzugeben, unter einem spezifischen Blickwinkel vielleicht sogar als „Hof-

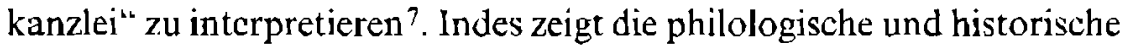
Analyse des Terminus é-dub-ba-a eine ganze Reihe von Ungereimtheiten, die sich nicht ohne weiteres hinter der scheinbar unverfänglichen babylonischen Übersetzung hït tuppi $(m)$ und schon gar nicht hinter einer kulturgeschichtlich so bedeutsamen Institution vermuten ließen, ohne deren Tätigkeit die Altorientalische Philologie um vieles ärmer wäre.

Schon A. Falkenstein notierte in seinem 1948 erschienenen Aufsatz „Der „Sohn des Tafelhauses“" die, wie er sich ausdrückte „merkwür-

wird im Zusammenhang des Rätsels nicht restlos klar. Rcal-bildlich könntc diese Aussage mit der aus den aB Terracotta-Reliefs bekannten "Göttin auf Gans" (+ Podest) in Verbindung zu bringen sein (vgl. zu diesen Darstellungen R. Opificius, UAVA 2, 80 ff; 211 f.). Aut der metaphorischen, in diesem Falle vermutlich bedeutsamen Ebene ist vielleicht der Zusammenhang mit einer sog. Gewichtsente herzustellen, deren Maßgeblichkeit bzw. Normcharakter als Tertium comparationis gedient haben dürfte.

${ }^{5}$ Dieses Wunschdenken von Lehrern und Absolventen wurde natürlich nicht unwidersprochen hingenommen und über das Bild des Welpen. der, blind zur Welt gekommen, ersi nach und nach die Konturen seiner Umgebung zu erkennen vermag, problematisiert. Vgl. hierzu Anm. 129.

6 E. I. Gordon, ВiOr. 17 (1960) 142; W. Heimpel, Tierbilder, 415f.: A. Sjöberg, AS 20, 159; W. Röllig, Neues Hundb. der Lit.wiss. 1 (1978) 24 (Ühersetzung); M. Civil, AulOr. 5, $18 \mathrm{tf}$.

7 F. R. Kraus, Vom mesopotamischen Menschen ... (1973) 25, hat außerhalb des Zusammenhanges dieses Rätsels eine Identität von postulierter Hofkanzlei und $\dot{e}-\mathrm{dub}$ ba-a vermutet, jedoch ausdrücklich vermerkt, daß die Probc aufs Exempel aufgrund der Lücken unserer Kenntnis nicht durchzuführen sei. 
dige Pleneschreibung" in é-dub-ba-a, vertraute sich am Ende aber doch der babylonischen Übersetzung bït tuppi $(m)$ "Tafelhaus“ an ${ }^{8}$. Eine Reihe von tastenden, aber auch phantasievollen Versuchen, jeweils nur in Anmerkungen versteckt ${ }^{9}$, zeigt die ganze Problematik, die sich hinter dem auf $/-b a /$ folgenden $/-a /$ verbirgt ${ }^{10}$. Einigkeit besteht heute zumindest darin, daß in é-dub-ba-a keine Genitivverbindung (,Haus der Tafel(n) ...") vorliegt" ${ }^{11}$, die zumindest auf syntaktischer Ebene dem babylonischen bit țuppi $(m)$ entspräche. Insbesondere nach der Analyse von A. Cavigneaux ${ }^{12}$ kann es als wahrscheinlich gelten, da $\beta$ der sumerische Terminus é-dub-ba-a eigentlich als é $d u b$ ba-a "Haus, das Tafeln zuteilt" zu verstehen ist ${ }^{13}$.

Nicht minder bemerkenswert ist die Geschichte des Terminus édub-ba-a. Er findet erstmalige Erwähnung in einer Hymne des Königs Sulgi von Ur (2094-2047), der hier von sich behaupten läßt, er habe als Kind zwei dieser Einrichtungen mit besonderem Erfolg besucht ${ }^{14}$. $\mathrm{Daß}$ es Unterweisung und Schule, die sich eines weitgehend festgelegten Curriculums bediente, jedoch schon weit über tausend Jahre früher gegeben hat, beweist nicht nur die Existenz der lexikalischen Texte der Uruk IV-Zeit 's. Der Zusammenhang von Schriftlichkeit und Schule als Schriftzeichen und deren Inhalte vermittelnde Institution hat von Anbeginn des Schreibens an als Voraussetzung zu gelten. Die ersten Belege für den Beruf des Schreibers als (hoher) Funktionär in Tempel oder Palast stammen aus dem frühen

8 Vgl. A. Falkenstein, WO 1 (1948) 174-175 „é-dub-ba-a weist ... die merkwürdige Pleneschreibung $[/-b a-a /]$ auf".

9 So hat z. B. B. Landsberger, zitiert von A. Sjöberg, AS 20, I, Anm. 1, die These vertreten, die Schreibung $\dot{c}-d u b-b a-a$ sej sozusagen als Kunstwort eingeführt worden, um sie von dem älteren (!?) é-Dus(=kišib)-ba „Lagerhaus" zu unterscheiden.

$10 \mathrm{Vgl}$. D. O. Edzard, AfO 23 (1970) $92 \mathrm{f}^{5}{ }^{5}$ : „Es fragl sich, ... ob é-dub-ba-a überhaupt von Hause aus etwas anderes als "Haus der Tafeln' bedeutete". Eine ganz. andere Sicht der Dinge vertritt W. W. Hallo. Fs. A.. Sjöberg (1989) 236: „Perhaps he $[\hat{A}$. Sjöberg] will accept the etymology ..House of the A-tablet".

11 Vgl. Civil (apud Hallo, Fs. Sjöberg. 23723): ,the expression [é-dub-ba-d] is not a genitive".

12 Vgl. A. Cavigneaux, Die sum.-akk. Zeichenlisten (1976) 8I: „Nebenbei kann man fragen, ob nicht dieses Verbum [i. e. /ba/] in dem Wort é-dub-ba-(a) vorliegt, d. h. ,Haus, das die Tafel ..."

13 Vgl. Edzard (zitiert von C. Wilcke apud Hallo, Fs. Sjöberg. $237^{2}$ ): „House which distributes the tablets”. $Z u$ fragen wäre indes, ob nicht (eher?) eine Deutung ..Haus, in dem Tufeln zugeteilt werden" in Betracht zu ziehen ist. Endlich wäre auch dic Analyse von /ba-a/ zu diskutieren: Etwa ba-e(d) $>$ ba-a(d)? [Hinweis C. Wilcke].

14 Vgl. hierzu K. Volk, Saeculum 47 (1996) 202f. mit Anm. 148.

is Darüberhinaus die zahlreichen Schülertexte späterer Perioden des III. Jahrtausends wie etwa diejenigen aus Fâra. Abu Salabihn, Girsu. 
III. Jtsd. ${ }^{16}$; der Name einer spezifischen (Aus-)Bildungseinrichtung, mit der jener verbunden gewesen sein könnte, jedoch nicht. Eben diese Situation finden wir auch in den Perioden nach dem Zusammenbruch des altbabylonischen Reiches, in denen seltene Erwähnungen des édub-ba-a ${ }^{17}$ bzw. des bĩt tuppätíl8 oder der mär ețuppe $\tilde{e}^{19}$ nicht mehr als Reminiszenzen ${ }^{20}$ an eine vergangene Epoche $7 \mathrm{u}$ sein scheinen ${ }^{21}$. Schließlich wird in den Literaturkatalogen des I. Jahrtausends kein

${ }^{16} \mathrm{Vgl}$. neben dem in der, Names and Professions List' aus Teil Abũ Șalābihn, Z.9 genannten dub-sar ,.Schreiber" auch den hierarchisch höher angesiedelten sa ğga (5. R. Biggs, OIP 99. 33 mit Anm, 29; 63; s, auch ED lú B (i) I [MSL 12, 13]; ED lú E. Z. 2 [MSL 12, 17]), der den schriftkundigen Tempelvorsteher bezeichnet. Es ist zu vermuten, daß die ersten Schreiber - im allerdings überproportional gut dokumentierten mesopotamischen Süden - in der Tempeladministration zu suchen sind.

17 Vgl. Sjöberg, AS 20, 160.

in ND 1120 r. 16-23 (Iraq 14 [1952] 69; vgl. H. Hunger, Kolophone [1968] 96, Nr. 314; Datierung: 9. Jahr Sargons). Der Kolophon führt einen unbekannten Stadtschreiber [PN] Iupsar āli auf, der sich auf eine lange Ahnenreihe, alles Stadtschreiber, beruft, an deren Beginn ein sonst nicht bezeugter Etel-pī-Marduk, 'útuB.SAR É tup-pa-a-ti „Schreiber des Tafelhauses" (welches?) steht. Hunger, op, cit., 9, notiert diesen Kolophon als singulär.

$10 \mathrm{Vgl}$. G. Çağirgan/W. G. Lambert, JCS 43-45 (1991/93) 90 f,; 93.

20 Besonders zu beachten ist in diesem Zusammenhang, daß ein altbabylonischer Edubba'a-Brief (VS 17, 44) im brt rēš in Uruk in seleukidischem Kontext aufgefunden wurde (vgl. hierzu J. Oelsner, Materialien [1986] 179). Weiterhin ist auf das jüngst von A. Cavigneaux, Fs, H. Limet (1996) l] ff. veröffentlichte Bruchstück CBS 1642 (neuassyrische Schrift!) zu verweisen, das in face B, Z. 2' ein é-dub-[ba-a ...] neben der zuvorgenannten nam-d[ub-sur ...] erwähnt. Der Text gehörte möglicherweise einem Beschwörungspriester aus Assur (vgl, op. cit. 11).

21 Dazu gehört auch die Existenz der sog. Examenstexte. die zwar das (vor-) altbabylonische Edubha'a terminologisch reflektieren, ansonsten aber alle Anzeichen von sekundär redigierten Werken aufweisen. Entsprechend sind die ältesten Textzeugen an den Beginn des I. Jtsds. zu stellen (vgl. Sjöberg, AS 20, 160). - Anders als Sjöberg. AS 20. $159 \mathrm{f}$. sehe ich keinen Gegensatz von altbabylonischem Edubba'a und ,privater", nachaltbabylonischer Schreibertradition. Die Untersuchungen von Wilcke, Isin Išān Bahrīyāt III (1987) 83, und D. Charpin, Le clergé (s. unten), zu den Tafelfunden in einzelnen Häusern von Isin bzw. Ur, wie auch die Tatsache, daß sich der berühmte Esağil-kīn-apli auf Asalluhi-manšum, einen ,Weisen‘ aus der Regierungszeit Hammurapis, zurückführt, zeigen, daß dic Tradition von, privatem Unterricht mindestens in die altbabylonische Zeit, sicherlich sogar auf dic Anfänge des Schreibens zurückgeht. Von Anfang an haben im Rahmen der Amtsnachfolge Schreiberfamilien, wenn nicht -dynastien existiert, seien sie nun Angestellte von Palast oder Tempel. Jedenfalls fand Unterricht sowohl in der Ur III- wie in altbabylonischer Zeit auch in einem Kontext statt, der nicht unter dem Signum é-dub-ba-a gehandelt wurde. Der Unterschied dürfte u. a. darin bestanden haben, daß diesen Ausbildungsstätten kein um-mi-a "Meister" vorstand, sondern lediglich ein dub-sar ,Schreiber". 
einziges von den großen Literaturwerken auf ein É-dub-ba-a zurückgeführt ${ }^{22}$.

Immer wieder in der Geschichte der Altorientalistik hat man geglaubt, die Institution Schule auch dinglich in Form eines charakteristischen Gebäudes nachweisen zu können. Als vor wenigen Jahren G. Farber einen Text veröffentlichte, der, wie sie annahm, „notwendige Arbeitsgänge beim Bau eines Schulhauses “ beschrieb ${ }^{23}$, schien der Beweis erbracht, daß Schulhäuser gezielt und systematisch gebaut wurden. Indes handelt es sich bei dem in diesem Text genannten é-dub. ba nicht um ein é-dub-ba-a, sondern um ein é-kišib-ba „Lagerhaus“, das als Teil eines é-amar-ra „Kälberhauses“ ja auch wesentlich plausibler wird ${ }^{24}$. Auch die aufgrund ihrer suggestiven „Schulbänke" 25 einstmals als solche klassifizierte „Schule" im Palast des Zimrilim in Mari ${ }^{26}$ konnte diesem Anspruch auf Dauer nicht gerecht werden $^{27}$. Die Örtlichkeiten, an denen nachgewiesenermaßen sowohl Schülertafeln, als auch ein Querschnitt des bekannten zeitgenössischen

$22 \mathrm{Vgl}$. W. G. Lambert, A Catalogue of Texts and Authors, JCS 16 (1962) $59 \mathrm{ff}$. In diesem Kontext ist hervorzuheben, daß die legendären Autoren/Redaktoren ausschließlich sog. Priestertitel führen (Lambert, op. cit., 74f.). Unklar bleibt der Zusammenhang von Taqīsa-Gula, dem berühmten Gelehrten und „Klagepriester" aus Nippur. und Šulgi in KAR 385 r. 44-45.

$23 \mathrm{Vgl}$. G. Farber, Fs. Sjöberg, $137 \mathrm{ff}$.

24 Entsprechend ist A 2976 r. 15 (von Farber, Fs. Sjöberg, 138 f. als é-dub-ba s̀à éamar-ra-ka gelesen und „(für) die Schule, die im ,Kälberhaus' gebaut worden ist“ übersetzt) zu revidieren.

25 Auch E. Stone, SAOC 44 (1987) 57, zu TA, Level XI, Floor 2, zählt die in locus 192 (Hof) festgestellten Bänke zur Grundausstattung (,necessary furniture“) dieser ,Schule“: "Benches against the walls provided the teacher and the pupils with places to sit".

26 Vgl. A. Parrot, MAM Il. Le Palais - Architecture (1958) 186 ff., "Les écoles" und pl. XLI-XLII, Rüume 24 und 25.

27 Kritisch hatte sich schon S. N. Kramer, History Begins at Sumer ${ }^{3}$ (1981) «8"), geäuBert. H. Waetzoldt, Keilschrift und Schulen in Mesopotamien und Ebla (1986) 39. formuliert seine Einschätzung wie folgt: „Er (der Ausgräber) fand dort aber keinerlei Unterrichtsmaterial, also besonders Schreib-Übungen und Tontafeln, sondern nur ovale Schüsseln, die kleine Muscheln enthielten. Sowohl die Größe der Räume (ca. 95 und $39 \mathrm{~m}^{2}$ ), die türlos miteinander verbunden sind, und die Anordnung der Bānke, als auch die Anordnung der Fundplätze und der Inhalt der Schüsscln sprechen m.E. eindeutig gegen die Deutung des Ausgrübers" (Parrot, MAM II (s. Anm. 26), 186, Anm. 3, hatte mit Verweis auf Syria 17 (1936) 21 notiert, in den genannten Räumen sei «matériel scolaire» gefunden worden). Wilcke, Isin - Iš̃an Bahriyăt III, 83, macht insbesondere die mangelhaften Lichtverhältnisse in den $50 \mathrm{~g}$ Schulräumen im Palast des Zimri-Lim in Mari gegen eine Identifikation der Räume als "Schulrāume" geltend. Festzuhalten ist weiterhin, daß die Edubba'a-Texte an keiner Stelie von Bänken sprechen, duf denen Schülcr hätten Platz nehmen sollen. 
Curriculums gefunden wurde, erwiesen sich als architektonisch wenig spektakuläre Räume in Tempeln ${ }^{28}$, Palästen ${ }^{29}$ oder weitaus häufiger als Privathäuser, u. a. von Priestern. Gut untersuchte Beispiele bieten das Haus des $\mathrm{Ku}$-Ningal und seiner Söhne in der sog. "Quiet Street" Nr. 7 im altbabylonischen $\mathrm{Ur}^{30}$, das Haus (aus dem Bereich TA) F-1, Level XI im altbabylonischen Nippur ${ }^{31}$, das Haus des Opferschauers Asqudum im sog. ,chantier $\mathrm{A}^{\prime}$ in Mari ${ }^{32}$, das des Rab'ānu in Ugarit ${ }^{33}$ oder das Haus des gala-ma ha „Oberklagepriesters" Ur-Utu in Tell edDêr, das den Hof dieses Hauses als Ort des Unterrichts ausweist ${ }^{34}$. Dieser Befund läßt sich denn auch direkt mit den Aussagen der Edubba’a-Texte verbinden: „Sagt der ,Mann des Hofes“: „Schreibt!", dann

i8 Zu den altbab. Schülertexten aus Uruk vgl. A. Falkenstein, BaM 2 (1963) 41. Anm. 185.

${ }^{24}$ So z. B. im Sîn-kāšid-Palast im altbab. Uruk: vgl. Falkenstein. BaM 2, 41.

${ }^{30}$ Die berühmte ,Schule' Broad Street No. 1 hat sich als Fehlinterpretation erwiesen. Zwar zeigt der reichhaltige Tafelfund in Broad Street No. 1 einen ausgezeichneten Querschnitt des Curriculums der altbabylonischen Zeit auf, einschlicßlich Rätseln und Edubba'a-Literatur (Katalog der Texte bej Charpin, Le clergé, 439-447): doch stammen offenbar alle Tafein aus dem Abfall (wic meist in Nippur; cf. Th. Jacobsen, AJA 53 [1957] 126a) und gehen damit auf eine unbekannte Ausbildungsstätte (frühere Besiedlung in Broad Street No. 1?) zurück (vgl. Charpin, op cit., 482 ff.; 485) Demgegenüber zeigl das Gcbäude Quiet Street No. 7 einen an Umfang und Breite bescheideneren Querschnitt einer privaten Ausbildungsstätte, die von dem Reinigungspriester Ku-Ningal und seinen Söhnen geleitet wurde. Zum Katalog der hier, teilweise im Zusammenhang eines Regals aus Lehmziegeln (Raum 6) gefundenen Texte vgl. Charpin, op. cit., 35-41; zur Beschreibung des Gebäudes sowie einer Analyse der Fundsituation vgl. Charpin, op. cit., $28 \mathrm{fr}$.

31 Vgl. kritisch Charpin, RA 84 (1990) 4 ff. zu (TA) F-i (= floor l) Level XI, wonach sich aufgrund der veröffentlichten Urkunden kein spezifischer Besitzer bzw. ummia namhaft machen lässt; vgl. weiterhin Charpin, RA 84, 6 ff. zu F-2, Niveau X. In dieser Phase, nach vorhergehender Zerstörung (Samsu-iluna 11), war offenbar Ninurta-rīm-ili der letzte Besitzer. Es bleibt eher unwahrscheinlich. daB das Gebäude in dieser Phase noch zu Unterrichtszwecken genutzt wurde. Neben dem genannten wurden auch in einer ganzen Reihe weiterer Gebäude Schülertafeln gefunden.

$32 \mathrm{Zu}$ den hier gefundenen Schülertafeln vgl. Charpin. Le clergé. 486; ders., M.A.R.I. 4 (1985) $453 \mathrm{ft} ; 462$.

31 Vgl. J. Krecher, UF 1 (1969) 134 ff. (mit älterer Literatur) zu den einzelnen Gattungen von Schülertexten, dic in den ,Archives de Rap’anu' gefunden wurden.

34 Vgl. M. Tanret, Akkadica 27 (1982) 47. Im genannten Hof (Chantier E, locus 4) fand sich auch ein zu $2 / 3 \mathrm{im}$ Boden eingelassenes Becken (cd. $80 \times 70 \times 35 \mathrm{~cm}$ ) aus gebrannten Ziegeln (à $34 \times 34 \times 7,5 \mathrm{~cm}$ ), in dem Ton zur Tafelherstellung stets feucht gehalten werden konnte, gleichzeitig aber auch abgearbeitete Übungstexte aufbereitet und der Wiederverwendung zugeführt werden konnten. Zum archäologischen Kontext s. insbesondere $H$. Gasche, Mes. Hist. and Environment Mem. I (1989) 19-20 mit pl. 9 . 
setz(t)e ich mich an meinen Platz. Meine Tafel hatte ich erhalten, die Vorzeichnung (des Meisters) war zu meinen Füßen ${ }^{35}$ eingeritzt" ${ }^{\text {"36. So }}$ schlüssig sich diese Darstellung gibt, so schwierig bleibt eine allgemeingültige Analyse angesichts der letztlich disparaten Fundsituation der Schülertafeln ${ }^{37}$, die in großer Zahl auch außerhalb des aufgezeigten Kontextes gefunden wurden, vor allem auf Straßen ${ }^{38}$. Auch die in diesem Zusammenhang stehende Frage nach den zum Schreiben notwendigen Lichtverhältnissen und den damit zu fordernden Räumlichkeiten ist nicht abschließend geklärt ${ }^{39}$.

Grundsätzlich wird man aber davon ausgehen können, daß Schule überall dort stattfinden konnte, wo sich ein Schreiber fand, der seinen

35 Diese Textstelle (s. folgende Anm.) könnte den Schluß nahelegen. daß im Bereich des Elementarunterrichtes Zeichenbeispiele durch den Lehrer im Sand des Hofes bzw. zu Füßen der am Boden sitzenden Schüler vorgegeben wurden (vgl. hierzu die Übersetzung der Zeilen durch M. Civil in: Anchor Bible Dictionary II [1992] 304 sub G.). Unabhängig von dieser Textaussage kam auch Tanret. Akkadica 27, 49, zu dem Ergebnis. daB aufgrund der Fundsituation im Haus des Ur-Utu in Tell-edDerr (s. vorige Anm.) Zeichenvorgaben ,dans le sable de la cour" gemacht wurden. Wesentlich wahrscheinlicher ist jedoch, daB die vom Lehrer vorgeschriebene Tafel „Zu Fũßen“ lag und der am Boden sitzende Schüler scine ihm zuvor zugeteilte unbeschriebene Tafel nach dieser Vorlage zu beschreiben hatte. Die beschriebene Szene kann in Verbindung gebrache werden mit der von D. O. Edzard apud C. Wilcke, Das Lugalbandaepos (1969) 168, vorgeschlagenen Deutung von Lugalbanda II, Z. 122 múrgu-zu dub-sar-sar-re-me-en als ..(hinsichtlich) dein(es) Rücken(s) bist du einer, der dauernd Tafeln schreibt", womit auf eine gebückte Haltung gleich derjenigen des Schülers angespielt wird.

36 Der Sohn des Tafelhauses, Z. 31-32 lú-kisal-lả-ke 4 sar-ra-ab-zé-en ù-bí-dull

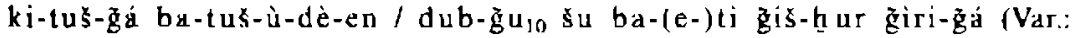

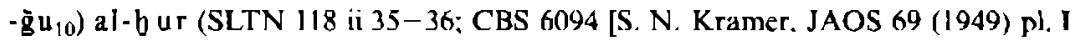
(nach 214)] i 22' I= Z. 31]; N 3565 [op.cit., pl. III] 3'-4': 3 N-T 905, 210 [SLFN, pl. 46] r. 2'-3' (unveröffentlichte Quellen nach Ms. M. Civil]).

3) Vgl. zu dieser Einschätzung Tanret, L'apprentissage de l'écriture à Sippar-Amnānum à la fin de la période paléo-babylonienne. in: (ed.) K. De Clerck/H. Van Daele, Fs. R, L. R. Plancke (1981) 31: „Nous estimons en effet quil serait vain dans leétat actuel de nos connaissances, d'essayer de procéder déjà à de grandes synthéses, alors que des études synchroniques et géographiquement limitées n’ont pas encore été entreprises".

38 Diese Feststellung beruht u. a. auf einer noch unveröffentlichten Untersuchung von C. Wilcke, welche die Fundsituation von Schul- und Altagstexten in lsin und Nippur zum Teilthema hat.

${ }^{39} \mathrm{Zu}$ fragen wäre hier. inwieweit sich der im Text (s. o.) genannte Hof eignet; denn das hier einfallende diffuse Licht ist für das Schreiben zweifellos nicht von Vorteil. Die von Wilcke, Isin - ls̆ăn Bahriyăt IIl, 83, diskutierte These, nachdern ,jeder Schüler an einem Fenster mit von links einfallendem Licht plaziert sein" müBte. findet jedoch einstweilen keinen Widerhall in den uns erhaltenen Edubba'a-Texten. 
Sohn ${ }^{40}$, hinwieder aber auch fremde Kinder ${ }^{41}$ in die Materie seines Berufes einführte ${ }^{42}$. Folgen wir den Aussagen der Schulstreitgespräche, so konnte er im Edubba'a zumindest durch einen Schreibergesellen (šeš-gal), vielleicht auch durch weiteres Personal unterstützt werden $^{43}$. Nach einer mehrjährigen ${ }^{44}$, genau gestaffelten Grundausbil-

40 Eın besonders schönes Beispiel bietet eine Siegelinschrift aus Keš (?) / Tell al-Wilăyà (i 1-2 und ii 3-4): Su-Ma-ma dub-sar / dumu Ś-Éšar um-mi-a (Sumer 16 [1960] nach 92, Tf. 6,1 [arab. Teil]). Leider ist m. W. nicht bekannt, ob der Schreiber Su-Ma-ma späterhín selbst um-mi-a "Meister“" geworden ist.

41 Vgl. etwa BM 78332 (CT 2, pl. 11; s. R. Frankena, AbB 2, 48 Nr. 81) r. 29--31 a-na

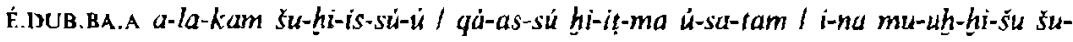
$k u-u n$,Lehre (meinen Sohn) zum Edubba'a zu gehen! Prüfe seine Hand! Lab ihm Hilfe angedeihen!" Beachte, daß nicht nur Findelkinder (Ai 7 iii 7 ff.; [MSL I, 99 f.]) sondern auch Ausländer, wie z. B. Kinder von versklavten Kriegsgefangenen eine Ausbildung zum Schreiber aufnehmen konnten (s. H. Waetzoldt, Das Schreiberwesen in Mesopotamien nach den Texien aus neusumerischer Zeit, unveröff. Habil.Schrift, Heidelberg 1974, 118). Zwar sind eigentliche Lehrverträge mit Schreibern bis heute nicht bekannt, doch setzt z. B. der Birašenna-Brief (zumindest für den kanaanäischen Bereich) eine dreijührige Lehrzeit voraus, wobei die Eltern des Schülers den Lehrer angemessen, d. h. entsprechend einer Vereinbarung, zu entlohnen hatten (vgl. A. Demsky, Fs. P. Artzi [1990] 165-170). In einen Lehr-Kontexc (wahrscheinlich) außerhalb des Edubba'a weist schließlich der Bricf aus Isin IB 1541, 6' 7': 11'-13' um-ma at-la-ma a-na-[k]u í-wa- $\{i-d a-k a]$ / ú-ra-ab-bi-ka ù $a-b a-k[a$ a-ta-

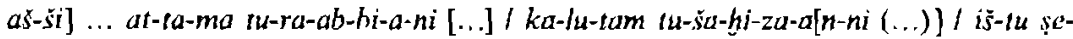
eh-re-ku a-d $[i \ldots]$..FolgendermaBen (schriebst) Du: ,Ich habe [dich] ,geschaffen', dich großgezogen, sogar deinen Vater [habe ich unterhalten'] ... Ja, du hast mich großgezogen, [...]; du hast mich den Beruf des gala-,Priesters' gelehrt. Seit ich klein war, bis [in mein Mannesalter(?) hinein ...]" (vgl. Wilcke, ZA 75 [1985] 189 f.). Dieser Brief zeigt, daß eine Lehrer-Schüler Beziehung über das reine Lehren (šühuzum) hinaus bestimmt sein konnte durch das "Schaffen", i.e. Formen (wulludum; vgl. Anm. 137) und Großziehen (rubbûm), und zwar von Anbeginn an (išru şęhrēku).

42 Mic dieser Beschreibung der mesopotamischen Gegebenheiten ist man rahe dem Befund. den die "Schule“ im alten Israel bietet, in den Worten von J. Barr: „The term school seems to imply some sort of institution with some continuity and some identity of membership" (BiOr. 40 [1983] Sp. 140, Rezension zu A. Lemaire, Les écoles et la formation de la bible dans l'ancien Israel = OBO 39 [1981]).

43 Die zahlreichen, insbesondere in der Schulsatire "Der Sohn des Tafelhauses" genannten Personen sind - auch angesichts der bisher bezeugten eher kleinen Schulen - keinesfalls repräsentativ. aber auch keine reine literarische Fiktion. Der um-mia ,Meister". auch ad-da-é-dub-ba-a „Vater des Tafelhauses" genannt, übernahm in der Praxis offenbar verschiedene Funktionen, die im Erzählzusammenhang als Bezeichnungen verschiedener Amtsträger erscheinen. Vgl. hierzu jetzt auch N. Veldhuis, Dutch Studies in Near Eastern Languages and Literature 2 (1996) 12, der ebenfalls nur zwei hauptamtliche Personen annimmt.

44 Nach Danel 14--5 konnten junge Männer nach einer dreijährigen Ausbildung in Schrift und Sprache der Chaldäer in den königlichen Dienst eintreten. Wenn es sich dabei um eine Grundausbildung handelt, so könnte diese Zahlenangabe - mit allem 
dung ${ }^{45}$ folgte eine Phase der Praxisorientierung und Spezialisierung. Die meisten von ihnen füllten am Ende ihrer Ausbildung durchschnittlich bezahlte Posten in der Verwaltung aus ${ }^{46}$. Manchen diente die Schreiberausbildung als Sprungbrett für eine Karriere als Funktionär in der königlichen Verwaltung ${ }^{47}$ oder an einem Tempel. Nur wenige widmeten sich auch nach dem Abschluß ihrer Ausbildung neben der Bewahrung der literarischen Traditionen dem literarischen Schaffen, konzipierten Königshymnen ${ }^{48}$, politische und religiöse Propagandaliteratur oder waren, wie etwa der berühmte Esag̃il-kĩn-apli (am Ende des II. Jtsds.), für die Kanonisierung einzelner Literaturwerke verantwortlich ${ }^{49}$. Besonders erfahrene Schreiber trugen schließlich den Ehrentitel um-mi-a ..Meister" ${ }^{\circ 0}$. Über sie, ihre Schüler und Gesellen

Vorhehalt und unter Berückșichtigung der Verhältnisse in neubabylonischer Zeit auch auf die frühere (und au(lerbabylonische) Schreiberausbildung angewandt werden (vgl, hierzu auch Anm. 41).

45 Vgl. hierzu Veldhuis (wie Anm. 43) $17 \mathrm{ff}$.; vergleichbare Ergebnisse für den Unterricht der neu- bzw. spätbabylonischen Zeit präsentierte P. Gesche in ihrem unveröf fentlichten Rencontre-Beitrag (Prag), betitelt "Was unterrichteten sic wirklich?" (mir von der Autorin in dankenswerter Weise zugänglich gemacht).

4h Zur Entlohnung von Schreibern in der Ur III-Zeit vgl. Waetzoldt (wie Anm. 41) $111 \mathrm{ff}$. Als .Anfangsgehalt' werden 60 Liter Gerste/Monat, in der zweiten Gehaltsstufe 120 Liter gezahlt ( 300 I Gerste bzw. 300 I Datteln entsprechen I Schekel Silber). Mit steigender Leistung und steigendem Verantwortungsbereich konnten bis zu 5000 Liter Gerste/Monat erreicht werden. Zusätzlich zur Enthohnung mit Gerste können Rationen von Mehl, Öl, Datteln, Wolle und Stoffe und das Angebot eines Versorgungsfe]des hinzukommen. $\mathrm{Zu}$ erwähnen sind weiterhin zusätzlicher Reiseproviant sowie .,Lohnfortzahlung im Krankheitsfall“(!), in Form von tüglich 2 Litern Gerste. Zur Entlohnung von Schreibern in anderen Epochen vgl. Waetzoldt (wie Anm. 4l) 113 ff. In allen Perioden sind starke Schwankungen zu verzeichnen, die im Zusammenhang mit der jeweiligen ökonomischen Gesamtsituation stehen. Nach der Höhe der Rationen und der Auflistung in Wirtschaftstexten wurden dic Schreiber zu den Handwerkern gezählt (gleich den ,Lchrern' der Elementarschulen im alten Griechenland), besaßen andererseits aber gesellschaftlich hohes Ansehen.

47 Entsprechend qualifiziert konnte er u. a. den Titel dub-sar-mah - lugal ..Oberschreiber des Königs" beanspruchen; vgl, Waetzoldt (wie Anm. 41) 57;81.

48 Vgl. hierzu M.-C. Ludwig, Išme-Dagan (1990) $41 \mathrm{fr}$.

$49 \mathrm{Vgl}$. I. L. Finkel, Gs. A. Sachs (1988) 143 fr. Esag̈il-kīn-apli war nach Auskunfı von BM $41237+$, Vs. 25' (I. L. Finkel, op. cit., 148 f.) für die Zusammenstellung der kanonischen Version des Handbuches für den Beschwörer SA.Gis = sukikkû verantwortlich.

so Wir wissen nicht, wann und aufgrund welcher Verdienste man sich einen solchen Ehrentitel zulegen durfte; vielleicht wurde er in einem bestimmten Alter oder nach einer gewissen Anzahl von Berufsjahren verliehen. Dic Tatsache, daB die in Urkunden der Ur III-Zeit crwähnten ummias (,Mejster', nicht des Edubba'a!?) wiederholt als Ernteschätzer in Garten und Feld verzeichnet sind, muß aber wohl so gewertet werden, daB sie nur aufgrund langjähriger Berufserfahrung zu einer solch verant- 
wird in einer Reihe von höchst aufschlußreichen und überaus lebensnahen Streitgesprächen aus der altbabylonischen Zeit berichtet. Als Ort des Geschehens wird mit Ausnahme der Streitgespräche zwischen 2 Frauen stets das É-dub-ba-a genannt. Textzeugen zu diesen Streitgesprächen fanden sich u.a. in den o.g. Privathäusern ${ }^{51}$. Man studierte dort also derlei Texte. Ob jedoch alle diese Lokalitäten, wiederholt Priestern gehörige Privathäuser, É-dub-ba-a genannt werden dürfen, ist $\mathrm{m}$. E. eher unwahrscheinlich; denn es scheint, als seien die Edubba'a genannten Institutionen, einst zumindest von Šulgis Vorgänger Ur-Nammu vor einem individuellen ökonomisch-politischen Hintergrund eingerichtet ${ }^{52}$ und in altbabylonischer Zeit in voller Blüte stehend ${ }^{53}$, ein spezifischer Fall von „Schule“, indem sie ganz auf die Bedürfnisse des Palastes ausgerichtet waren ${ }^{54}$. Dic auffallend säkulare

wortungsvollen Tätigkeit geeignet erschienen. Zu Belegen der Ur III-Zeit vgl. Wactzoldt (wie Anm. 41) 367.

s) So etwa die Nummern 3-NT 900 bis 901, die in Haus F. floor 1. Raum 205, level XI gefunden wurden, u. a. "Streit zweier Schulabsolventen" (3 N-T 900,15+; $3 \mathrm{~N}-\mathrm{T}$ 900,17); „Der Sohn des Tafelhauses“ (3 N-T 901,54: 3 N-T 901.55). Vgl. J. Heimerdinger, SLFN, vii.

S2 Duß es nicht Šulgi selbst ist, auf den die Erstgründung eines Edubba’a zurückgeht, ergibt sich aus seiner Schilderung. er habe als Kind zwei derartige Einrichtungen besucht. Inwieweit ,Neugründungen' in der Regierungszeit Sulgis eher ökonomisch oder mehr politisch motiviert waren, läßt sich nicht zweifelsfrei crkennen (vgl. zur Gesamtproblematik H. Waetzoldt, JAOS 111 [1991] 638 ad P. Steinkeller, SAOC $46^{2}, 17$, Nr. $6-8$ ). Nach dem historischen Gesamtkontext ist davon auszugehen, daß die Palastverwaltung zunehmend mehr Schreiber benötigte und diese auch ausbildete. Mit den politischen Veränderungen in der Folge der Akkadzeit dürfte auch das überkommene Curriculum in den als ideologisch zentral angeschenen Punkten reformiert worden sein. um Wertvorstellungen des Ur III-Königtums insbesondere über die schreibende Zunft gezielt zu verbreiten (vgl. C. Wilcke: Politik und Literatur im älteren Babylonien, in: Anfänge des politischen Denkens in der Antike, in: Schriften des Historischen Kollegs, Kolloquien 24: (ed.) K. Raaflaub [1993] 66 f.) Was Sulgı selbst anbetrifft, so scheint er, zumindest was dic Etablierung der ihm gewidmeten Hymnen im Schulbetrieb angeht, direkt in das traditionelle Curriculum eingegriffen zu haben. Seine Behauptung, traditionelles Gut der religiösen Kultur besonders intensiv gepflegt zu haben (Šulgi B 270 ff:; vgl. hierzu Ludwig, IšmeDagan 48 ff.), nährt den Verdacht gezielter Einflußnahme. Unübersehbar ist weiterhin, daB die Edubba'a-Texte (als Teil eines Pensums, das unter den Bedingungen schwindenden sumerischen Muttersprachanteils steht) so gut wie keine religiös bestimmten Wercvorstellungen reflektieren.

53 Vgl. Kraus (wie Anm. 7) 21 ff.

${ }^{54}$ Es ist mir kein Ur lII-zeitlicher oder altbabylonischer Beleg bekannt, in dem ein édub-ba-a im Zusammenhang eines Tempels genannt wird. Dagegen macht Kraus (wie Anm. 7) 23 ff., den Zusammenhang zwischen é-dub-ba-a und Palast deutlich. Vgl. schon A. Falkenstein. WO 1 (1948) 185 f. Das von Waetzoldt (wie Anm. 4l) 50 
Weltsicht ${ }^{55}$ der Schulstreitgespräche bestätigt diesen Befund auf seine Weise.

In den Schulstreitgesprächen erfahren wir nun nicht nur von der Örtlichkeit an sich, den handelnden Personen, dem schulischen Pensum, von der Selbstdarstellung des einen und der beißenden Kritik am anderen, mehr oder weniger erfolgreichen Schreiberschüler ${ }^{56}$. Wir erhalten darüber hinaus einen in der Literatur einmaligen Einblick in die Methoden, mit denen man die Heranwachsenden innerhalb dieses Kontextes zu erziehen versuchte.

Auch den Ummias des beginnenden II. Jahrtausends war klar, daß der fromme Wunsch, ein Schüler möge, wie es in dem oben zitierten Rätsel so schon heißt, ,geöffneten Auges" das Institut verlassen, nicht ohne ihr didaktisches, aber auch disziplinarisches Zutun erreicht werden konnte. $\mathrm{Zu}$ viele Versuchungen konnten ein Kind von dem von den Erwachsenen vorgegebenen Weg, der an fest definierten Leistungsmaßstäben orientiert war, abhalten. So machten die Meister Lob (wie zu allen Zeiten) davon abhängig, inwieweit das von ihnen diktierte Pensum erfüllt und die Verhaltensvorschriften des Edubba'a eingehalten wurden. Ernüchternd überdies, daß, wie die Edubba’a-Komposition "Der Sohn des Tafelhauses" dies sarkastisch schildert. Lob nicht selten nur durch gezielte ,Geschenke" an den um-mi-d ...Meister" orwirkt werden konnte. Neben der strengen Zurückhaltung beim Loben

für die Ur III-Zeit reklamierte *é-dub-ba-énsi „Tafelhaus des Stadtfürsten" ist, wie schon von A. L. Oppenheim, AOS 32. Tf. 12: 6ff. A 4 Vs. 2 notiert. ein ékišib-ba-énsi "Lagerhaus des Stadtfürsten“.

ss Hierfür spricht einmal der Tenor der Werke, der auf „einfache" Schreiber herabsieht und soziale und ethische Werte völlig losgelöst von jedem religiösen Hintergrund bestimmt. Weiterhin aber vor allem die Tatsache, daß nach einem der bedeutendsten Edubba'a-Briefe (s. J. van Dijk, Or. 58 [1989] 449 ff.) der Unterricht bei einem privaten Lehrer in dessen Haus (é-vm-mi-a-ka) als, nach Ansicht des Absenders, wenig schön (igi-g̈á nu-sa, "meiner Ansicht nach nicht schön“), als nicht der Bezeichnung é-dub-ba-a würdig angesehen wurde. Das é-um-mi-a ist also é-dub-baa-gi-na in-nu ..kein rechtes Edubba'a“.

56 Die ,weniger erfolgreichen' Schüler, die nicht die letzte Stufe des Edubba'a erreichten. geben sich vermutlich insbesondere in Urkunden zu erkennen, in denen sie $u$. a. konventionelle Zeichenformen durch ,homophone' ersetzen (z. B. lú : Iu: pà : pa), glossieren (lugal : lugal|ll), silbisch wiedergeben (hu $\tilde{\mathrm{g}}: \mathrm{hu}$-ù $\tilde{\mathrm{g}}$ ). Zeichen aufgrund graphischer (sa $10:$ gaz; da : á) oder lautlicher Ähnlichkeit ( $z \dot{a}: z a)$ verwechseln. auslassen (z. B. lú in im-ma - - bi >-me) oder standardisierte diri-Komposita verandern (tuk umbi [šU.ĞAR.TLR.LÁ.BI : ŠU.ĞAR.TLR]) bzw. durch ,homophone Zeichen-

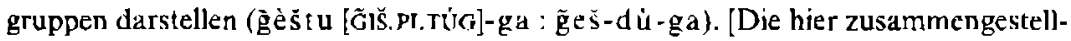
ten Beispiele gehen auf eine unveröffentlichte Sammlung einschlägiger Belege von C. Wilcke zurück]. 
hat man immer wieder versucht, die Kinder mit lebenden Vorbildern wie etwa den Eltern oder erfolgreichen Geschwistern zu konfrontieren und damit zu motivieren. Nicht selten zielte dieses Vorhaben ins Leere, ebenso wie der Versuch, den Schülern Orientierungshilfe durch das Studium der Taten der Heroen der epischen oder hymnischen Literatur $^{57}$ zu geben, die ja Bestandteil des Curriculums waren ${ }^{58}$. Vielleicht gelang dies eher über den Wettbewerb mit Gleichaltrigen, mit denen man auf einer anderen Ebene der Auseinandersetzung stand, als mit den Erwachsenen. Mit diesen mag sich manch ein Schüler ebenso wenig wie mit den Inhalten des Unterrichts identifiziert haben. Das immer wieder in Aussicht gestellte Sozialprestige und die Verlockung mit Wohlstand könnte schließlich den einen oder anderen am Ende doch dazu angehalten haben, seine Aufgaben wenigstens soweit als existentiell wie sozial nötig auszuführen. Kaum ein Kind dürfte schließlich seine Kindheit ohne Ermahnungen und Appelle verbracht haben. Halfen auch diese nichts, so konnte es schon einmal Schläge setzen, wobei in der privaten Sphäre immer wieder ausdrücklich auf Schläge verzichtet $^{59}$ und der Sinn solchen Tuns durchaus reflektiert wurde ${ }^{60}$. Daß andererseits die Schuloberen gegenüber den ihnen anvertrauten Kindern wenig Sentimentalität an den Tag legten, überrascht nicht weiter.

$57 \mathrm{Zu}$ den edukatorischen Aspekten der Tradierung von Königshymnen und Briefen vgl. Wilcke (wie Anm. 52) 63 ff. Hervorzuheben ist die Darstellung Šulgis, u.a. in Śulgi B, der von der Schule über den Sport, von jagdlichen (als erzicherische Vorstufe zum Soldatentum) und militärischen Aktivităten bis hin zur Musik alles nur in Perfektion zu beherrschen vorgab. Vergegenwärtigt man sich dic Rezeption dieses Werkes und seiner Themen im Curriculum des altbabylonischen Schulwesens, so ist ein Vergleich mit klassischen ,Bildungsromanen“ wie etwa Xenophons ,Kyropaideia“ keineswegs abwegig.

58 In diesem Zusammenhang zu vergleichen ist die Funktion der altgriechischen epischen Literatur im Rahmen des Unterrichts. E. A. Havelock, Preface to Plato (1963) 47, schreibt hierzu: „It follows that the history of Greek poetry is also the history of early Greek paideia. ... Epic had been par excellence the vehicle of the preserved word throughout the Dark Age. At that time it must also have been the main vehicle of instruction".

59 Vgl. K. Volk, Saeculum 47 (1996) 196 ff. mit Anm. 117 und 119. Nachzutragen ist an dieser Stelle Ai VII iii 17 te-bi nu-ub-ra-ah / le-e|s-su ul im-ha-as] „Er (der Adoptivvater) schlug seine (i.e. des Adoptivkindes) Wange nicht". Die Tatsache, daß diese Wendung in das standardisierte Formular übernommen werden sollte, zeig1, daß 3 der Schutz nicht-leiblicher Kinder gegenüber Auswüchsen der patria potestas als Anliegen des, Geselzgebers' galt.

60) "Der Vater und sein mißratener Sohn" 62 sìg-ge-dè sìg-ge-dè zi ba-ir šu bae-ri-bar "(Dich) zu schlagen (und noch einmal) zu schlagen, da wurde ich nur betrübc (Deshalb) habe ich Dir freien Lauf gelassen" (vgl. Volk, Saeculum 47, 197 mil Anm. 120). 
Unerlaubtes Reden, zu spät Kommen, unerlaubtes Aufstehen, Verlassen des Edubba'a, Trinken, akkadisch reden oder eine miserable Handschrift, das waren nur einige der Gründe, Schüler durch Schläge zurechrzuweisen. Als Maximalstrafen schrieben die Statuten des Édub-ba-a schließlich zweimonatigen Hausarrest und Fußfesseln vor.

So vergleichsweise leicht und sicher sich die Methoden beschreiben lassen, mit denen man in altbabylonischer Zeit Kinder zu erziehen versuchte, so schwierig wird das Terrain, wenn man versucht, die vermittelten Inhalte und Werte festzuhalten, zumal diese ja durchaus auch als Reflexe des Gesellschafts- und Menschenbildes des bürgerlichen oder, in unserem Zusammenhang präziser, des städtischen Milieus der altbabylonischen Zeit zu gelten haben. Als Kraus, der sich intensiv mit den Menschen des alten Babylonien und ihren Lebensbedingungen beschäftigt hat, in seinem bekannten Werk ,Vom mesopotamischen Menschen der altbabylonischen Zeit und seiner Welt" (s. Anm. 7), schrieb: „Wie nicht anders zu erwarten, finden sich, soweit ich weiB. keine ausdrücklichen Aussagen über Werte und Ideale in der Literatur", so überging er dabei stillschweigend die, wenngleich aufgrund der damaligen Überlieferungslage noch begrenzte, so doch weitreichende Diskussion eben dieser Fragestellung durch van Dijk in seinem „L'É dubba et son Ésprit" aus dem Jahre 1953. Van Dijk hatte seinerzeit sogar schon die Frage angeschnitten, wozu die Schulstreitgespräche, die, wie er feststellte, Inhalte und Werte vermitteln ${ }^{61}$, gedient haben könnten, gelangte allerdings zu keinem schlüssigen Ergebnis. Ausgehend von einer deutlich verbesserten Materialbasis ${ }^{62}$ kann nunmehr der Versuch einer Analyse unternommen werden, da sie als Voraussetzung für das Verständnis dieser Texte zu gelten hat.

Zwar erklärt sich kein einziges der Schulstreitgespräche in dem Sinne, $\mathrm{da} ß$ es seine eigene Existenz definierte. Dies ist nach dem kulturgeschichtlichen Zusammenhang kaum überraschend. Und doch erhellen die Aussagen der auf uns gekommenen Texte den historischen Hintergrund und seine Protagonisten auf eine ganz bezeichnende Weise. So charakterisieren die Schulstreitgespräche die handelnden Personen als Menschen, die aus einem nichtsumerischsprachigen Umfeld stammen ${ }^{63}$, für die Sumerisch eine zu erlernende Fremdsprache war. Die Tatsache,

6) Vgl. zu diesem Ansatz auch B. Alster, Fs. Lokkegaard (1990) 8, der zu „Zwei Frauen“ (B) ausführt: „One cannot deny that the text sets a moral code ..."

62 Hier nicht mitgerechnet sind die Examenstexte.

${ }^{63}$ Dieser Hinweis ergibt sich u. a. aus BM 54746 (vgl. Civil. Fs. R. Borger [1998] 1 ff.). da der Lehrer den Schüler zunächst vom Akkadischen in das Sumerische übersetzen läBt. 
daß man Prügel erhalten konnte, wenn man unerlaubterweise Akkadisch sprach, oder daß die Protagonisten der Schulstreitgespräche sich gegenseitig vorhalten konnten, ihre Zunge sei für das Sumerische mit seinem völlig anderen Lautstand gegenüber der Muttersprache gänzlich ungeeignet ${ }^{64}$, spricht für sich. Wenn es nach den Schulstreitgesprächen weiterhin ein erklärtes Lernziel war, sumerische Konversation zu beherrschen ${ }^{65}$, so mu 8 gefragt werden, wie dieses Ziel in einem Umfeld erreicht werden konnte, welches das Sumerische nicht mehr als überall frei erlernbare Alltagssprache kannte. Weder die Elementarübungen noch die zahlreichen lexikalischen Listen und Formulare für die verschiedenen Sparten, selbst die Literatur eignete sich nur bedingt zu solchem Zweck ${ }^{66}$. Hingegen erlaubten die schon früh im Unterricht eingesetzten Proverbien ${ }^{67}$ als Fragmente von Umgangssprache und vor allem die Dialoge das Einüben von Alltagskonversation. Dies zeigt jedoch nur den formal-didaktischen Aspekt ${ }^{68}$, denn den Lernenden wurden über

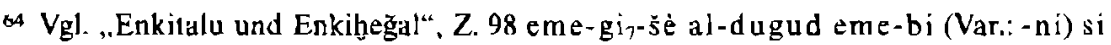
nu-ub-sá ,Was das Sumerische anbetrifft, so ist er schwerfällig. Jene Zunge kommt nicht zurech" [Var.: „Seine Zunge"] (TMHNF 3,42 ii 13 [vgl. Wilcke, Kollationen, 38, aủ Kolumne ii]; Ni. $4114+4139$ [ISET 2, 108) r. 3: Var. nuch unpubl. Texizeugen, Ms. Civil).

${ }^{65}$ Vgl. „Edubba'a Regulations" (s. Civil, Fs. M. Birot [1985] 69), Z. 4 [eme]-'gi ${ }^{7}$ ta injm e-da-bal-e-en "Auf sumerisch kann ich mich unterhalten". Auch eine Überseczung .Aus dem Sumcrischen kann ich übersetzen“ ist möglich, doch scheint nach dem Kontext die erstgegebene Deutung plausibler. Andererseits ließe sich die zweite Möglichkeit an BM 54746 (s. oben, Anm. 63) anbinden.

66 In der Schule der Humanisten wurden nach dem Auswendiglernen von einzelnen Wörtern (parallel zum Memorieren ganz kurzer, auf die allerwichtigsten Erscheinungen beschränkter grammatischer Regeln) wührend der Lektüre klassischer Schriftsteller "kurze bemerkenswerte Sätzchen aus den gelesenen Texten" auswendiggelernt (s. A. Bömer, Die lateinischen Schülergespräche der Humanisten I. Auszüge mit Einleitungen, Anmerkungen und Namen- und Sachregister. Quellen für die Schul- und Universitätsgeschichte des 15. und 16. Jahrhunderts [1897] 3).

67 Vgl. hierzu Volk, Saeculum 47, 186, Anm. 47; N. Veldhuis (wie Anm. 43) 20. Insbesondere Sprichwörter, die sich mit Tieren beschäftigen, haben hier einen doppelten didaktischen Wert: Der Schreiberschüler (dub-sa r-cu r) wird nach dem intensiven Studium von Listen nicht nur erstmals an kleine, vollständige Sä ze gewöhnt (vgl. hierzu auch H. Vanstiphout, JCS 31 [1979] 126); darüberhinaus wird er in diesen Sprichwörtern mit archetypischen Verhaltensweisen konfrontiert, die, weil im Bereich der Tierwelt angesiedelt, auf diese Weise ,kindgerecht" vermittelt werden können.

v. Dazu gehört u.a. auch die Pllege der Dialoge zum Erlangen einer professionellen Streitfähigkeit vor Gericht. Diese Ansicht wird insbesondere bestärkt durch „Zwei Frauen“ B, Z. 184-187 (TIM 9, 6:13-18//31-38), vgl. hicrzu Alster (wie Anm. 61) 8 mit Anm. 17. Von Interesse ist in diesem Zusammenhang die Bedeutung, die man den Disputationen im Schulbetrieb in den Statuta Collegii Sapientiae (als éin historisches Beispiel) beimaB. Danach galt die Disputation als „Feile des Verstandes“ (cum dispu- 
diese Schulstreitgespräche gleichzeitig auch ganz dezidierte Ansichten über Mensch und Gesellschaft vermittelt. Die angesprochenen Themen reflektieren nämlich sowohl Verhältnisse des schulischen wie auch und insbesondere des täglichen Lebens: Ein den Schülern geläufiges Milieu, in dem sie sich selbst wiederfinden konnten. Aber es sind nicht die Gedanken der Schüler, die hier artikuliert werden, sondern diejenigen ihrer Lehrer, die zum Ausdruck brachten, was sie selbst bewegte und was sie vermitteln wollten, auch wenn einzelne Themen, zumindest nach unserem heutigen Geschmack, nicht unbedingt für Heranwachsende geeignet schienen. Die um-mi-a „Meister“ sind es schließlich, die als unantastbare Autorität über den Dialogen stehen.

Wenn auch die große zeitliche Differenz einen Vergleich eigentlich verbietet, so sei dennoch darauf verwiesen, daß es die mittelalterlichen Humanisten waren, die, geistesgeschichtlich mit einer analogen Situation konfrontiert, indem sich das Lateinische nur noch als eine degenerierte Verkehrs- und Literatursprache darstellte, auf die Wirkung der Schülergespräche (Collocutiones) ${ }^{69}$ zur Revitalisierung der reinen lateinischen Sprache in Wissenschaft und Alltagsleben setzten und gleichzeitig als Dialogschreiber bzw. Redaktoren alten Stoffes ihre Weltsicht dem Munde der debattierenden Schüler entspringen ließen ${ }^{70}$.

tacio lima sit intellectus „Da die Disputation die Feile des Verstandes ist“), die es im Haus der Weisheit regelmäBig zu pflegen gelte. Die Scholaren seien zum Argumentieren verpflichtet, „bei Wahrung einer gewissen Bescheidenheit in Worten, Gebärden und Stimme, - zu dem Ende. daß durch Disputieren aus gegensätzlichen Meinungen die handgreifliche Wahrheit herausgeschält wird" (vgl. J. Kerer, Statuta Collegii sapientiae - Satzungen des Collegium Sapientiae zu Freiburg i. Br. 1497: FaksimileAusgabe [Lindau-Konstanz 1957] 85f.). Unter "handgreiflicher Wahrheit“ (veritas manifesta) verstand man natürlich Konformität entsprechend den herrschenden Lehren.

69 Vgl. insbesondere die collocutiones duorum puerorum de rebus puerilibus ad invicem loquentium, ein zweisprachiger drastischer Dialog zwischen den Schülern Syriscus und Petrelius, der deutliche strukturelle und gar phraseologische Parallelen zu einigen sumerischen Strcitgesprächen aus altbabylonischer Zeit aufweist. So beginnt dieser Dialog mit den Worten: Unde venis, Petrelle? ... Quid illic fecisti? (vgl. „Zwei Frauen“ [B], Z. I me-ta-àm àm-di-di-in „Woher kommst du?"*; ,Der Vater und sein mißratener Sohn“, Z. 1-3 'me-ta-àm'à m-du-un /'me-sé-àm al-du-un/ me-še-àm i-du-dè-en ,Woher kommst du? Wohin gehst du (gerade)? Wohin wirst du noch gehen?"“). Der Text erscheinr als Anhang zu ciner aus den ersten Jahren des 16. Jahrhunderts stammenden, von dem Zwoller Rektor Herrmann Torrentinus besorgten, erklärenden Ausgabe von Hymnen und Sequenzen. Vgl. Bömer (wie Anm. 66) I. 67 ff. Noch das im 18. Jhdt. von Franz Xaver Brixi (1732-1771) verfaßte Werk „Der Schulmeisterista“ (Erat unum cantor bonus) verwendet Floskeln, die sich nahezu wortgleich (kontextverschieden, jedoch, auf das Genre bezogen, archetypisch) schon in sumerischen Streitgesprächen finden.

$30 \mathrm{Vgl}$. Bümer (wie Anm. 66) I. 5. 
Doch zurück in die Schule der altbabylonischen Zeit. Zu welchem Zweck, so fragt man sich zum Beispiel, dienen die Streitgespräche zwischen Frauen als Unterrichtsstoff, wo doch der Anteil an Frauen unter der Gilde der Schreiber marginal scheint, wo die in den analogen Streitgesprächen unter jungen Männern üblichen Diskussionen um Pensum und schulische Erfolge schlichtweg inexistent sind und das Betreten des Edubba'a (nach Textvarianten aus dem Streitgespräch zwischen „Zwei Frauen“ [B]) möglicherweise den Herren der Schöpfung vorbehalten war? ${ }^{71}$ Es konnte weder nur der Dialog im Emesal, der, Frauensprache', gewesen sein noch die reine Übung zu forensischen Zwecken. Es dürfte vor allem ein ganz spezifisches Frauenbild im Vordergrund gestanden haben, das den Schülern als positiv und erstrebenswert bzw. ablehnenswert und anstößig vermittelt wurde. Die Ansprüche waren dabei nicht gering. Als erste Voraussetzung galt, daß eine junge Frau aus seßhaften $^{72}$ und nicht abhängigen Verhältnissen stammen mußte ${ }^{73}$. Neben

71 Vgl. "Zwei Frauen“ (B), Z. 27 é-dub-ba-a du $u_{8}$-du $u_{8}$ /é-a sage-e mu-te-te „Das Edubba'a ,öfnend"; im Hause hast du dich den Sklaven genähert" [oder etwa te-

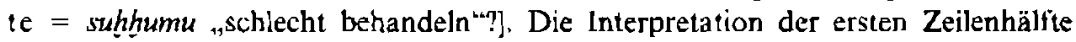
„Das Edubba'a ,öffnend" " folgt den unveröffentlichten Textzeugen (Ms. Civil). Die publizierten Textzeugen (VAT 12579 [=FAOS 2/1, Tf. 74] ii 7-8 bzw. Ni, 4509 [ISET 2, Tf. 22] Vs. 3 schreiben am Zeilenanfang jedoch lediglich é-vuB-ba, was sowohl als orthographische Variante $z u$ é-dub-ba-a anzusehen sein könnte, wie auch als bewusstes Wortspiel in der Lesung é-kisib-ba „Lager-/Schatzhaus". Danach wäre zu übersetzen: „Das Lager-/Schatzhaus ,öffnend“ “. d. h. das mit einem Siegel verschlossene Gebäude erbrechend. In diesem Zusammenhang ist zu beachten, daß das é-dub-ba-a in dem eingangs erwähnten Rätsel mit einer dub-šen .Schatzkiste" verglichen wurde. Die Interprezation der Zeile in ihrem Kontext bleibt vorerst jedoch unsicher, da aufgrund der unterschiedlichen Textüberlieferung auch andere Wortgrenzen in Betracht gezogen werden können. Zur Vorstellung, daß Frauen eine dem é-dub-ba-a, wenngleich nur anachronistisch vergleichbare Bildungseinrichturig wie das mittelalterliche Domus sapientiae nicht zu betreten hutten, vgl. den Abschnitt De conuersatione mulierum in domo sapientiae prohibita ,Vom Verbot des Umgangs mit Frauen im Haus zur Weisheit": „Wir wollen weder einer Frau noch Mädchen, welchen Stands sie auch sei, Gelegenheit geben, das Haus oder unsere Scholaren zu besuchen ..." (s. Kerer [wie Anm. 68] 72f.).

72 Vgl. „Zwei Frauen" (B), Z. 96a [ù za-e] lú irik $i^{k 1}$ tuš-ù-me-en / ù at-li ša a-li šu$\check{s u-b i}$..[Und du] willst ein Mensch sein, der die Stadt bewohnt (akk.: den man in der Stadt wohnen läBt)?" (RA 24 [1927] 36, Vs. 12/Rs. 12; s. E. I. Gordon, BiOr. 17 [1960] 141, Anm. 151); vgl. „Zwei Frauen“ (B), Z. 17 irikı rniggin kar [níg̃in]é-éa $\mathrm{ku}_{4}-\mathrm{ku}_{4}$, (ln) der Stadt umherziehend, (am) Hafen/Markt(?) [umherziehend), in (fremde) Häuscr eintretend" (VAT 12579 [FAOS 2/1. Tf. 74] i 28-29); vgl. hierzu „Zwei Frauen“ (B), Z. 136 šu-dag-dag-'ga' ha-la-ni "Umherstreifen ist ihr Los“ (UET 6/2,157:9).

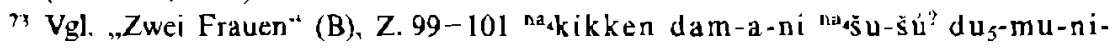

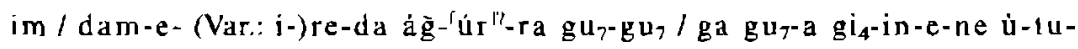


handwerklich-technischen ${ }^{74}$ und, was die gesamte Haushaltsführung anbetraf, organisatorischen Fähigkeiten ${ }^{75}$, die ein Mädchen von ihrer Mutter in Vollendung erlernt haben sollte, neben Sauberkeit ${ }^{76}$, ordentlicher Kleidung ${ }^{77}$, Pünktlichkeit ${ }^{78}$, Zuverlässigkeit ${ }^{79}$, Mäßigung ${ }^{80}$, Be-

da e-re-dè- (Var.: e-)ne „Der Mahlstein ist ihr Gatte, der Šu...-stein ihr Kind. Gattin eines Sklaven. Spelzen essend. Gesäugt von Mägden, geboren unter Sklaven“ (BE 31 42: 15-16; TCL 15,6 r. 23-25; VAT 12579 [FAOS 2/1, Tf. 75] iii 21-23: UET 6/2,158 r. 41-43; ein unveröffentlichter Textzeuge [Ms. Civil], liest in Z. 100 ág̀-ùr-ra). Zu Z. 101, vgl. Alster (wie Anm. 61) 8, Anm. 19.

74 S. unten, Anm. 89.

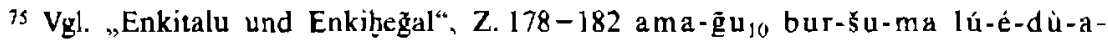
ka (Var. UET 6/2,153 r. 34; -kam) / géme-ta 10-àm igi-ni-šè al-sug-ge-es "Meine Mutter ist eine kluge Frau. die den Haushalt aufgebaut hat. Von den Mägden stehen 10 vor ihr“" (TMHNF 3,42 iii 21-22; STVC 133, Vs. ' ii l'-2'; UET 6/2.153 r. 34-35); vgl. zu diesen Zeilen Volk, Saeculum 47 [1996] 193 mit Anm. 89. Das Gegenteil dieser hier geschilderten Mutter wird dargestellt in "Der Streit zweier Schulabsolventen“. Z. 45 uma-zu-sè šu-bal-(a-)ak-àm „Was deine Mutter anbetrifft, so ist sie eine, bei der das Oberste zu unterst gekehrt ist" (CT 42,47 ii 2: CT 58,56:19'); vgl. auch „Zwei Frauen“ (B), Z. 42 a ma $_{5}$ nu-mu-unge-en é a-ráa-šè nu-mu-mar „Das Frauengemach machtesı du nicht zuverlässig, den Haushalt (hast Du) nicht verständig gegründet“ (BE 31,28:13): vgl. ebơ. Z. 135b 'ama ${ }_{5}-n i-s e ̀ ~ l a-b a-a b-d u_{7}$ "Für ihr Frauengemach ist sie ungeeignet" (UET 6/2,157:8).

${ }^{76} \mathrm{Vgl}$. „Zwei Frauen" (B), Z. 124 uḥ-ta zé [šà]-'ğar'-ta úš „Vor lauter Ungeziefer sich raufend, vor Hunger verendend" (unpubl., Ms. Civil).

77 Vgl. „Zwei Frauen“( (B), Z. 52 dam-zu túg nu-um-mu $u_{4}$ za-e "ugáḡ-dára' múmú „Dein Gatte trägt kein (anstündiges) Gewand, (und/[weil?]) du bringst Lumpen hervor" (BE 31,28 r. 1; SEM 114 r. 4; Ni. 4599 [ISET II 89] 8a).

${ }^{78}$ S. unten, Anm. 89.

${ }^{9}$ Vgl. ,Zwei Frauen“ (B), Z. 47 'lú' nu-kal-la munus nu-gi-na „Ehrlose Person. unzuverlässige Frau“ (VAT 12579 [= FAOS 2/1, Tf. 75] r. iii 7: SEM 114:4; BE 31,28:17).

$80 \mathrm{Vgl}$,Zwei Frauen“ (B), Z. 67 [ki]-še-er nu-luku na-á $\tilde{g}-$ munus-e la-ba-(ab-) $\mathrm{du}_{7}$, „Sie hat keine MäBigung, für Frauenangelegenheiten ist sie ungeeignet" (UET 6/2.158:20; VAT 12579 [= FAOS 2/1. Tf. 74] r. iii 29: Var.: ]a-ba-Dr; Ni. 4599 [ISET 2, Tf. 89] r. 5: Var.: nu-nuz für munus); vgl. weiter .Zwei Frauen“ (B). Z. 15 giri $i_{17-z a l}$-šè gub-ba' s̀à-gal-bi-eš ù nu-ku $u_{4}$,Die hochnäsig dasteht, (aber) wegen entsprechender (Sorge um) Verköstigung niche schlafen (kann)" (VAT 12579 [FAOS 2/1, Tf. 74] i 25-26); .,Zwei Frauen“ (B), Z. 133-134 nu-nuz-sun - na šu-

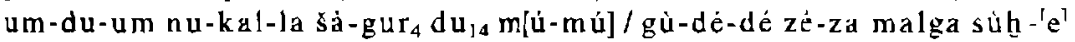
„Herrische Frau, die eine ehrlose ,Lippe hat, hochfahrend, beständig Streil anzertelnd, dauernd redend, aber an einem Sprachfehler leidend, von verwirrtem Verstand" (UET 6/2,157:6-7); das Nebeneinander von gù-dé-dé und ż́-7a legt ein Zitat aus der altbabylonischen Scrie lú $=., \bar{s}$ nahe, die nach den „Edubba'a Regulations", Z. 14 und „Der Streit zweier Schulabsolventen", Z. 4 zum Lehrstoff der Grundstufe gehörte; vgl. MSL 12, 162, Z. 138-139 lú-zé-Za =ha-su-ú/lú-gù[d] $\dot{e}^{-}-\mathrm{dé}=\check{s} a-\dot{s} s-s \dot{a}-a-\dot{u}$. 
scheidenheit ${ }^{81}$, Anstand ${ }^{82}$, korrektem Sozialverhalten ${ }^{83}$, dem Intriganz ${ }^{84}$ und Streitsucht ${ }^{85}$ entgegenstanden, maß man dem äußeren Erscheinungsbild ${ }^{86}$ ebenso wie den ,intellektuellen“ Fähigkeiten ${ }^{87}$ einer Frau erheblichen Stellenwert bei. Auf ganz bemerkenswerte Weise konnte gerade bei diesen Antipoden die im Alltag immer wieder als augenscheinlich empfundene Diskrepanz von Schein und Sein thematisiert werden. So heißt es in einem der Streitgespräche zwischen Frauen:

k) Vgl. "Zwei Friluen” (B), Z. 44 ta-(a-)a š šà-zu al-gur, ,Warum bist Du so überheblich?"* (BE 31.28:15; VAT 12579 [FAOS 2/1, TS. 74] ii 38; SEM 114:1).

R2 Vgl. „Zwei Frauen“ (B), Z. 31 téš nu-tuku duc-mu-mu-lu (Var.: lú)-nu-kal-la .Schamlose, Kind ehrloser Leute" (VAT 12579 [FAOS 2/1, Tf. 74] ii 13: Ni. 4509 [ISET 2, Tf. 22] 6; BE 31,28:1, Var.: téš nu-tuku mu-lu nu-kal-'́a' „Schamlose, Ehrlose"). Vgl. Alster (wie Anm. 61) 8, Anm. 19.

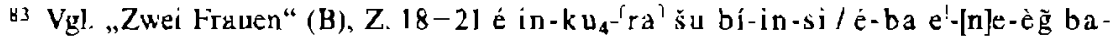
an'-tuku / dam dam-da im-ta-an-kúr / dus-mu ama-da im-da-ri „Das Haus, das sic betral, ,üllte' sje (umgehend) in (ihre) Hand. In jenem Hause hatte sie das. Wort" (oder: „bekam sie einen Rechtsstreit" [?]). Gatte mit Gattin verfeindete sie. Das Kind entriß sie der Mutter“ (VAT 12579 [FAOS 2/1. Tf. 74] i 30-34).

84 Vgl. „Zwei Frauen“ (B), Z. 181-182 a-na-aš-e dumu-munus'-lú tab-ba-zuum / in mu-un-dúb kar-kid ba-an-du 11 dam mu-un-taka $/ /$ am-mi-ni a-no ma-ur-ti a-ki-li-im tap-pá-a-ri-k[i] / pi-iš-lam 'tu'-up-pi-iš ha-ri-im-Ji ta-aq-bi-i mu-tam tu-še-zi-bi-lš (TIM 9,6:9-10//26-29) ,Warum beschuldigtest du die Tochter eines Mannes (..Bürgers"), deine Genossin, (indem) du sie eine Hure nanntesc (akk.: "Hure!' sagtesl) (und) ihn dazu brachtest, sich von seiner Gattin zu scheiden'?" Vgl. CAD 1101 s. v. harimtu, bil, section sowie Alster (wic Anm. 61) 8, Anm. 18; s. auch „Zwci Frauen“ (B). Z. 153 kar-k[id] 'éš'-dam-ma še-en-lul mu-du ${ }^{\prime}{ }^{\prime}-\left[d u_{11}\right]$ dam tuku" dam taka (Ni. 9730 [ISET I] Tf. 89] r. 2; UET 6/2.157 r. 25; CT 42,44:15-16a; Ergänzungen nach dem unveröff. Ms. von M. Civil) „.Hure, in der Kneipe verbreitete sie (fortwährend) Lügen: hat (?) sie cinen Ehegatten. scheidet sich der Gatte (gleich wieder) von ihr". Vgl. zur Zeile Alster (wie Anm, 61) 8, Anm. 18.

${ }^{4}$ Vgl. .Zwei Frauen“ (B), Z. 118 [murub ${ }_{4}$-bi-a] du ${ }_{14}$ àm-ma-ḡágá / i-na he-ri-risi-na sa-al-tam $i a-s ̌ a-a k-k a-n i$ „Unter ihnen (den Nachbarinnen) entfachst du Streit" (RA 24, 36:19: r. 20; Ergünzung nach Ms. Civil); ähnlich „Zwei Frauen“ (B). Z. 115 [usar usa]r-da du 14 mú-mú-ne / si-it-tam it-ti ši-it-tim tu-uš-té(=e)-șe-e-li (RA 24, 36:16; r. 17; vgl. C. Wilcke, ZA 59 [1969] 87, Anm. 90).

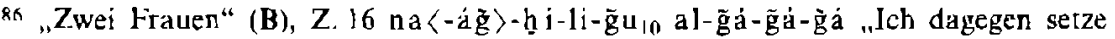
meinen Licbreiz/Sexappeal ein" (VAT 12579 [FAOS 2/l, Tf. 74] i 27). Daneben „Zwei Frauen" (B), Z. 139 i-bi-du 8 um-ma-ne mùs-me(-bi) gi ${ }_{4}$-in-'e-n[e] ..(Mit dem) Blick von (alten) Frauen. (dem verhärmten) Antlitz von Mägden“ (UET 6/ 2.157, u. R. 12: CT 42,44: I; vgl. hierzu Alster [wie Anm. 61] 8. Anm. 19).

87 ,Intcllektuelle' Fähigkeiten beziehen sich nach dem kulturellen Gesamtkontext nicht auf die Kenntnisse von Literatur, Kunst oder Philosophie. In welchem Maße derlei bei einer Frau des Alten Orients vorauszusetzen ist, bleibt im Einzelfall kaum nachvollziehbar. Es ist cher damit gemeint, daß eine Frau das Management der Familic und des Hauswesens im weitesten Sinne. das z. B. juristische wie ökonomische Kenntnisse voraussetzte, klug und geschickt (,weise') zu beherrschen hatte. 


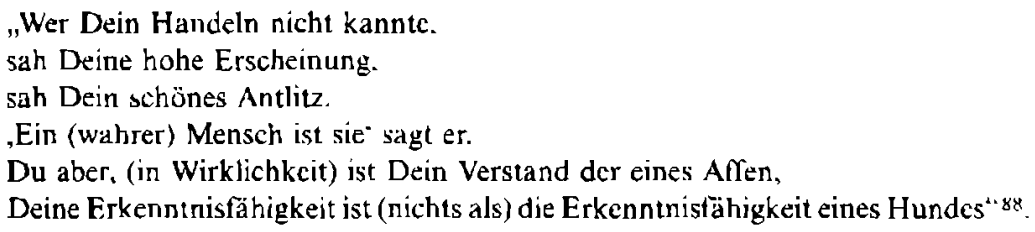

Die Streitgespräche zwischen Frauen, dic, wie schon betont, keinerlei schulisches Milieu reflektieren, behandeln mehr oder minder ausschließlich deren traditionelle Lebensbereiche. Nichts und niemand wird von der Kritik der Kontrahentin ausgespart. So heißt es etwa:

.Wolle kann sie nicht zupfen, mit der Spindel nicht spinnen. Die Hand taugt für die Arbeit nicht. Beim ,Eintreten' (zur Arbeit) und, Herausgehen' ist sic säumig“89.

Einem Schüler wurde anhand einer solchen Aussage vermittelt, daß eine von der Gesellschaft respektierte Frau in der Lage sein mußte, geschickt mit Wolle und Spindel umzugehen, aber auch pünktlich bei der Arbeit zu sein ${ }^{90}$. Besondere Aufmerksamkeit galt in den Streitgesprächen zwischen Frauen deren Verhalten in der Öffentlichkeit. So war eine Frau, von der man sagte:

„Die einen Mann bloBstellt, junge Frau. Lästerreden verbreitend: niederträchtige Reden führend: Provokantin, sich mit Fäusten schlagend; fluchend, verlogenc Worte wechselnd"9|

natürlich kaum das, was dic Pädagogen ihren Schülern als erstrebenswert empfahlen ${ }^{92}$. Bestimmte Verhaltensweisen wurden bei beiderlei

88 NBC 7805:25-30 (unveröffentlichtes Ms. Cavigneaux) lú níg-ak-Lu nu-un-zua/alan-sukud-da-zu igi i-ni-in-bar/müš-me-sat-ga-zu igi i-ni-in-bar/ lú-àm ib-bé / za-e dím-ma-zu dim-ma ugu"sua-b[i]/malga-zu malga-urgi $_{7}$-ra $x^{\top}[(x)]$. Vgl. zu diesem Text Volk, Saeculum 47, 191 mit Anm. 79.

89 Vgl. "Zwei Fraucn“ (B), Z. 68-69 siki nu-mu-un-da-peš $\sigma^{-c}$ हbala nu-muun-da-šir-šir $/$ kig̃-e šu nu-mu-un-da-sá k $u_{4}-k u_{4}$ è-dè ab-lá (unpubl, Ms. Civil).

90 Vgl. auch "Zwei Frauen" (B). Z.67 (s. o. Anm. 80).

91 „Zwei Frauen“ (B), Z. 57-59 lú sag̀-ir-ra nu-nuz sikil-dù-a / e-ne-èg-sig

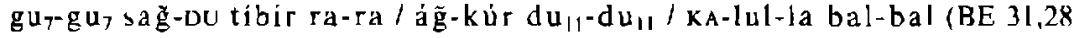
r. 29-3); SEM 114 r. 10-12; 3 N-T 918,444 [SLFN pl. 59] r. 4-6: 3 N-T 906,248:13: Ni. 4356 [ISETT II 9.3] 3-5].

92 Vgl. hıcrzu „Zwei Frauen“ (B), Z. 156-157 u[r] šu-zi-ga eger-mu-1u-ne-k[a ...J/

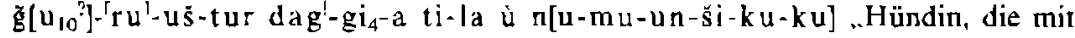
erhobener Pfote hinter den Männern [herläuft(?)], die jungen Männer. die in der Nachbarschaft leben. können ihrelwegen nicht schlafen" (CT 42,44, Vs. 18-19: Ergänzung der Verbalform von Z. 157 nach Z. 130 [s. dazu Volk, Saeculum 47, 191 mit Anm. 80]). 
Geschlecht permanent gegeißelt, so etwa das Herumtreiben auf dem Marktplatz, auf Straßen und Gassen. So heißt es immer wieder ${ }^{93}$ :

„Steh nicht auf dem Marktplatz herum! ${ }^{94}$

Treib Dich nicht auf der Straße herum!

Wenn Du durch die Gassen gehst, schau' nicht immer umher!"

Ebenso, wie man eine Frau von Ehre und Anstand mit einem Katalog von Eigenschaften charakterisierte, so konnte man auch einen Mann als solchen beschreiben. Zu diesem Zweck ließen die Ummias die Schüler in den Schulstreitgesprächen (und Schulsatiren) darüber debattieren, was denn einen Mann von geistiger Reife ${ }^{95}$, einen , wahren ${ }^{2}$ Menschen ausmache und welchem Procedere er sich zu unterwerfen habe, damit er als Mensch von Erziehung anerkannt werde und zu Ansehen und Reichtum gelange. Auch er sollte, als äußeres Zeichen seines Standes, seßhaft sein ${ }^{96}$, nicht in Abhängigkeit stehen ${ }^{97}$, unverschuldet ${ }^{98}$ und, noch besser, reich sein ${ }^{99}$,

93 Vgl „Der Vater und sein mißratener Sohn“, Z. 29-36 tilla nam-ba-e-gub-bu-dèen / sila-a nam-mu-nigin-dè-en /e-sír-ra dib-bé-da-zu-dè igi nan-barbar-re-en/hé-sung-ne-en ugula-a-zu ni háe-em-dè-te/ni-te-ní-te-a-zu-dè ugula-a-zu ki a-ra-an-ág/ù-mu hué-tuku lú-tab-ba-zu sá du ${ }_{11}-g a-n[a] / a-$ ga-aš nam-ba-an-na-gid-gi $i_{4}-d \dot{e}^{\prime}-[e n] / g i_{4}-m e-a s-z u-n e ~ s ̌ u ~ n a m-m u '-e-k a ́ r-$ kár-[(...)].

94 Auf dem Marktplatz zu stehen, galt auch bei (jungen) Frauen ais äu(3erst schlechtes Benehmen: $x$ dili-àm tilla $i-g u b-b u-[x] /$ in $i-g i g-g e_{4}$ hné-bi-du $u_{11}[x] \ldots$ allein stchst Du auf dem Marktplatz. Fürchterliche Schimpfworte hast Du ausgesprochen" (.Streit zweier Frauen“(A) NBC 7805:16-17, unveröf. Ms. Cavigneaux). Auch das Herumtreiben auf Straßen und Gassen galt offenbar als häufig kritisiertes Fehlverhal1en, auch bei den Frauen: e-sir-rà ù - ba -e-gub in a[1-dúb-dúb-bu] „Kaum bist du auf die Straße getreten, schon schimpfst du herum" (,Zwei Frauen" (B), Z. 70 [UET 6/ 2, 158:23: Ergänzungen nach Ms. Civil]): sila-a gub-gub e-sír-ra ni $\mathrm{i}_{10}-\mathrm{ni} \mathrm{i}_{10} / m u-1 a-$ $z i-z a-a t$ re-bi-a-tim sà-hri-ra-at su-qá-a-tim „überall in den Gassen stehend. sich auf den Straßen herumtreibend" / (akk.:) ,die in den Gassen steht, sich auf den Straßen herumtreibt" („Zwei Frauen“" (B), Z. III [RA 24, 36:13; r. 13; einsprachig sumerisch: BE 31,42, r. 31; CT $58,58: 16 a\})$.

95 Vgl. Volk, Saeculum 47, 194 mit Anm. 97-98.

os Vgl. „Der Streit zweier Schulabsolventen“, Z. 77 é nu-tuku e-sir-ra nú-nú „Ohne Haus, auf der Straße lagernd" (SLTNi. 113 r. 2; 3 N-T 919,461:2).

97 Vgl. „Der Streit zweier Schulabsolventen“, Z. 73 kiši $i_{4}(-a)-a k$ ğe ${ }_{26}$-nu lú-dabs kus-ta bar-bar "An ciner Kopfhälfte geschoren, Auf!, (Du) Häftling, aus dem Fremdland vertrieben" (SLTNi. 113:6; SLTNi. 116 r. 22; UET 6/2,155:20; Ni. 9850 [ISET I 142] r. 3).

$98 \mathrm{Vgl.} \mathrm{„Der} \mathrm{Streit} \mathrm{zweier} \mathrm{Schulabsolventen“,} \mathrm{Z.} 63$ níg-urs-ra (Var.: Ni. 9865:2 nígnu-ra) uh-uh-da ğen-na ur ${ }_{5}$-ra me-te-zu ,... mic Ungeziefer umherlaufend Zinsschulden (sind) deine Zier" (SEM 65:15; SLTNi. 116:12: UET 6/2,155:10; Ni. 9865 [ISET II 92]: 2).

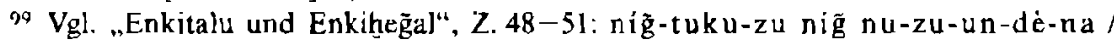

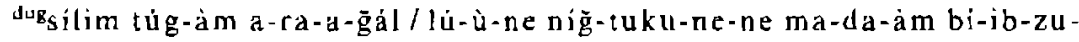


nicht arbeitsscheu ${ }^{100}$, säumig ${ }^{101}$ oder faul ${ }^{102}$, sondern strebsam ${ }^{103}$, gepflegt $^{104}$, geschickt ${ }^{105}$, zuverlässig sein ${ }^{106}$, Anstand - auch wenn

zu-ne / iri-me-y úku ù Jú-niğ-tuku g̃iskim an-tuku-uš „Dein Reichtum (ist) etwas, das wir nicht kennen. (Nichts als) ein silim-Gefäß mit einer Stoff(abdeckung) ist bei dir vorhanden. Die Leute lassen das (ganze) Land (= alle?) ihren Reichtum wissen. In unserer Stadt hatten Arme(r) und Reiche(r) äußere Erkennungszeichen“ (die Umschrift der Zeilen richtet sich im Wesentlichen nach unveröffentlichten Textzeugen [Ms. Civil]; teilweise erhaltene Textzeugen sind: TMHNF 3,42 i $27-28$ [dazu Wilcke, Kollationen 38; Z. 27 bleibt mir unverständlich]; UET 6/2 152:1: zu Z. 49 vgl. W. Sallaberger. Der babyl. Töpfer [1996] 106).

$100 \mathrm{Vgl}$. „Der Streit zweier Schulabsolventen“. Z, 103 kig̃-ta ba-zăh (-me-en) ganbaka mu,-mu, ba-gub-bé-en „Von der Arbeit entflohen, stehst du auf dem Markt. platz schreiend herum" (CT 42,47 r. iii 2; UET 6/2,156:6; TMHNF 3.43 r. iii i4).

$10 t \mathrm{Vgl}$, „Der Streit zweier Schulabsolventen", Z. 98-102 schildert nicht nur den bei der Arbeit Säumigen, sondern gibt ein insgesamt eindrückliches Beispiel für eine schlechte

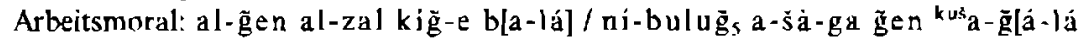

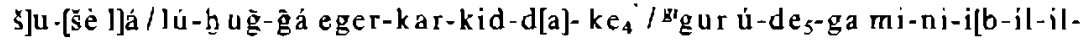
i] / $u_{4}-b u r u_{14}-k a$ á-zu šà-gal-zu [nu-ub-da-sá], ,Herumstreunend, trödelnd, bei der Arbeit ist er säumig. Prahlend, auf dem Feld umherziehend (und dabei) einen Ledersack bei sich (wörtl.: an der Hand) tragend. Mietling. Abhängiger einer Prostituierten. Im Korb trägt er gesammeltes Reisig: am Tag der Ernte kommt dein Lohn deinem Futterbedarf nicht gleich" (TMHNF 3,43 r. ii 9-13; UET 6/2,156:1-5. Ni. 4073 [ISET II] r. ii 4-7; die Ergänzung von Z. 99 folgt PSD A/4, 74; von Z. 100 PSD B 179 , mit Verweis auf die zweisprachige Parallele K 83.38, Z. 4-6 [s. W. G. Lambert, BWL. 255; C. Wilcke, Das Lugalbandaepos (1969) 156 mit Anm. 426]; zu Z. 102 vgl. G. Gragg, Sum. Dimensional Infixes [= AOAT 5. 1973] 58; die Ergänzungen von Z. 98 und 101 gehen auf einen unveröffentlichten Textzeugen [Ms. Civil] zurück).

$102 \mathrm{Vgl.} \mathrm{„Schreiber} \mathrm{und} \mathrm{Aufseher",} \mathrm{Z.} 34$ a-na-ẵ-àm lú-g̃á-la-dag-ga-gim g̀i š ma-ab-hur-bur-re-en. Warum zeichnest du mir wie einem Untätigen alle Vorgaben auf?" (HAV 19:11; SRT 28 r. 6). Vgl. ebda. Z. 37b u4-na-me níğ-gá-la-dagga-g̃ $u_{10}(-u s)$ sá ${ }^{\prime} a b^{\prime}-r a-\dot{a} m-m u-r i ̀-j b-d u_{11}$ (SEM 59:8; Var.: sá ba-ra-àm-muri-íb-du, [unpubl. Ms. Civil]; sá ma’-mu-ri-ib-du, [HAV 19 r. 14]) „War ich jemals untätig?" (wörtl: , "hat Dich meine Untätigkeit jemals erreicht?"/Var.: ,hat man Dich wegen meiner Untätigkeit jemals angegangen?").

${ }^{103}$ Als besonders eindrückliches Vorbild an Strebsamkeit in Verbindung mit schulischen Erfolgen läßt sich König Šulgi darstellen: „Als ich klein war, habe ich im Edubbàa auf den Tafeln von Sumer und Akkad die Schreibkunst erlernt. Keiner der Einheimischen schricb Tafeln so (gut) wie ich". Vgl. zu diesen Zeilen aus Šlgi B Volk, Saeculum 47, 202 f. mit Anm. 148.

104 Vgl. „Der Strcit zweier Schulabsolventen“, Z.47 luh (Var.: ki-de -de $_{\text {- }}$ ga luh

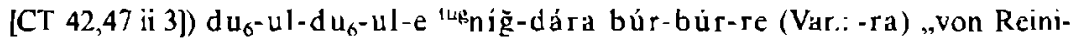
gung(sabfällen) (Var.: .,am Ort der Reinigung von Reinigungsabfällen) verkrustet. mit Lumpen bedeckt" (Ni. 9715 [ISET II 84] r. 6; CT 58,56:19': CT 42,47 ii 3); vgl. zu dieser Zeile PSD B 197 sub 2.2: s. weiterhin schon oben (Anm. 98) zu ebd. Z. 63 uh-uh -da g̃en-na „mil Ungeziefer herumlaufend".

$105 \mathrm{Vgl}$,Der Streit zwcier Schulabsolventen", Z. 79-80 dukútul-ar-za-na imšs-rin-

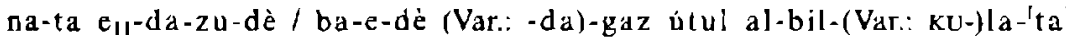


nicht naturgegeben ${ }^{107}-$, Ehrgefühl ${ }^{108}$ und gute Eßsitten haben ${ }^{109}$, gebildet ${ }^{110}$, maßvoll sein ${ }^{11}$, kollegial handeln ${ }^{112}$, Konflikte lösen kön-

,Wenn du den Topf mit Grütze vom Ofen herunterholst, zerbricht cr bei dir, weil der Topf erhitzt war “ (TMHNF 3.43 i 5-6; UET 6/2.155 r. 24-25; SEM 65 r. 89; 3 N-T 903,104 [SLFN, pl. 59] 4).

ion Vgl. "Enkitalu und Enkiḩeg̃al“. Z. 12 lú nu-me-en-na kíg-gi ${ }_{4}{ }^{-}$a [nu-ub-gi-na] „Der du kein Mann bist. (einer), der Arbeit nicht zuverlässig ausführt“ (ISET I 65 i 11; der Textzeuge ist nach weiteren unveröffentlichten [Ms. Civil] ergänzt. TMHNF 3.44 i 5 bietet folgende Variante [lú nu-me-e]n-na kíg-gi 4 -a nu-ub-gi $i_{4}^{-a}$.. Der du [kein Mann bist]. (einer), der (s)eine Arbeit nicht ausführt").

$107 \mathrm{Vgl}$, hierzu die ebenso nüchterne wie im Unterton zynische Formulierung in „Schreiber und Aufseher“, Z. 22-23 [ki-2a-za t]és-lú-ùlu-ka (Var.;-kam) ma h-bi gú huéri-dù/[ú-téš]-a-kam (Var.:-ka) šà ab-šed 4 (-dè) nam-tag-ga al-du $u_{g}$-e „Unterwürfigkeit zeigt den Arstand (wörtl.: ist der Anstand) eines Menschen. Soll(te) sie Dir ganz besonders verhaß̧t sein? Das Kräutlein (i. e. die Medizin) rür den Anstund ist sie. Sie kühlt das Gemüt (des Kontrahenten), löst (mögliche) Schuld". 2. 23, Var. -ka (wenn nicht rein orthographisch): „Im (d. h. durch das) Kraut des Anstandes kühlt er (= man?) das Herz, löst die Schuld" (Texte: Ni. 4243 [SLTN 114], side a' 25-26; TMHNF 3.37:3-4; Ergänzungen und Varianten nach Ms. Civil). Unterwürfigkeit wird auch in SP 5,92 als verhaßtes, hündisches Verhalten angesehen: u r-[g]im ki- $z a-$ za hul a -[a]b-gig "es ist (ihm?) verhaßt, sich wie ein Hund zu unterwerfen “. Bezeichnenderweise heißt es jedoch in den Schulstreitgesprächen, da $B$ vernünftig handle, wer cinem Vorgesetzten gegenüber respektvoll spreche: vgl. "Schreiber und Aufseher", Z. 29 dub-sar úmun-ak sunc-na(-bi) ugula-a-na (Var.: ugula-(a-)ni) mu(un-)na-ni-ib-gi $i_{4}-g i_{4}$..Der vernünftig handelnde Schreiber antwortet seinem Vorgesetzten demürig" (Ni. 9679 [ISET II 84] r. 7'; SRT 28 r. 1; TMHNF 3.37:13).

108 Vgl. „Der Streit zweier Schulabsolventen“, Z. 97 a-ga-ğ $u_{10}$-šè na-an-gub-bé-en

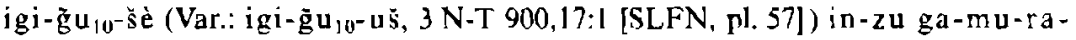
ab-[til] „Hinter mir sollst du nicht stehen! Meiner angesichtig will ich dir deine Beschimpfungen zu Ende bringen" (TMHNF 3,43 r. iii 8; $3 \mathrm{~N}-\mathrm{T}$ 900,17 [SLFN, pl. 57] 1: 3 N-T 923,503 [SLFN, pl. 56] r. 1/2; Ni, 4073 [ISET Il 87] r. iii 3; Ergänzung der Verbalform nach einem unveröffentlichten Textzeugen [Ms. Civil]).

in Vgl. "Enkitalu und Enkiȟeğal“, Z. 117-118 kaš un-nağ eme-sig al-gu ninda un-gu, si-sá-bi nu-ub-bé „Hast du Bier getrunken, redest du, nicder daher (oder: rülpst du(?); D. O. Edzard). Hast du Brot gegessen, kannst du nicht mehr recht reden" (TMHNF 3,42 ii 32-33; ISET 190 ii 9-10).

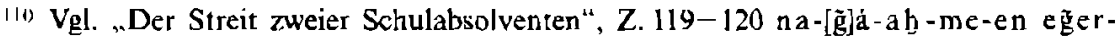
inim-'ma'-zu nu-zu / lú-sikil-dú-a-me-en níg-nam(-e) nu-(e-)zu .Du Trottel, die Bedeutung deiner Worte (wörth.: deine Rückseite des Wortes) erkennst du nicht! Du Lästerer, nichts weißt du!“ (Ni. 4207 [ISET II 86] r. 6-7; 3 N-T 900,15 + 3 N-T 900.27+ 3 N-T 901,57 [SLFN, pl. 57] r. 4-5: CT 42.47 r. 17-18]. Vgl. auch „Der Streit zweier Schulabsolventen“, Z. 76 ní-su-ub lú-IM dim-ma uku ugu $\mathbf{4}^{-}$bi .Ekstatiker, Idiot (mit dem) Verstand eines Affen" (SLTNi. 116 r. 25; SEM 65 r. 5; UET $6 / 2,155 \mathrm{r}, 1)$.

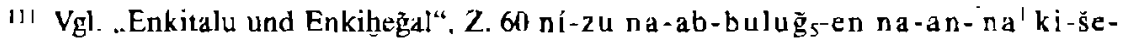
er [nu-e-tuku] „Du sollst Dich nicht selbst loben. Außerdem hattest Du kein Maß!" (UET 6/2,152:10; ISET I 65 ii 2: Ergärzungen nach PSD B 179 sub bu lugs). 
nen ${ }^{113}$ und sich vor allem niemals selbst loben ${ }^{114}$. Auch durfte sich glücklich schätzen, wer bereits auf eine etablierte Abstammung verweisen konnte ${ }^{115}$. Solch gegebenes Kapital konnte natürlich leicht verspielt werden. In jedem Falle mußte man sich als Kind in der hierarchischen Welt der Erwachsenen zurechtfinden und bewähren. In dem Schulstreitgespräch „Der Schreiber und sein Aufseher“ erzählt ein nunmehr arrivierter Aufseher eines privaten Haushaltes 116 einem ihm unterstellten jungen Schreiber aus seiner eigenen Schulzeit. Er kam, wie er sagt, zu Lob und Ansehen, weil er stets die Ratschläge seines Meisters befolgte. Als unabdingbare Voraussetzung galt es dabei, sich mit Ausschließlichkeit einer gestellten Aufgabe zu widmen ${ }^{117}$, pünktlich und

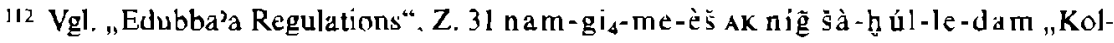
legialität zu pflegen isı etwas, das das Herz froh macht". Vgl. zu dieser Zeile C. Wilcke. ZA 25 (1969) 90. Zum kollegialen Verhalten außcrhalb des Bereiches Gleichgestellter vgl. jetzt A. Cavigneaux. Fs. H. Limet (1996) $20 \mathrm{f}$. zu CBS 11945 (neubab.!). r. 17-18// VS 10 ii 8-9; Ni. 4193 (ISET I 136) r. 11.

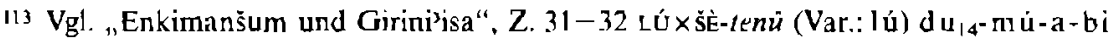

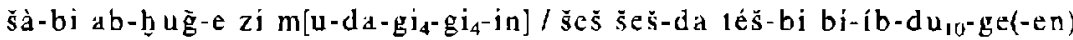
ṡà m[u-da-šed $\left.{ }_{4}-d \dot{e}-e n\right]$ „Das Gemüt von Gefangenen, (unter denen) sich Streit erhoben hat, beruhige ich, Aufruhr kann ich umwandeln: Bruder mit Bruder versöhne ich vollständig, das Gemüt kann ich kühlen “ (UET 6/2.150:31-32; 3 N-T 916,34l [SLFN, pl. 60]: 3"'-4"; Ergänzungen nach unveröffentlichten Textzeugen [M5. Civil]).

114 Vgl. „Enkitalu und Enkiheğal”, Z. 57-58 tukum-bi ni-zu ár-šè ba-AK(-x") / à r AK-dè-e la-ba-ab-d|u $\left.{ }_{7}{ }^{2}-u_{n}\right]$,Wenn du dich selbst lobst, bist du nicht gecignet, gelobt zu werden" (UET 6/2,152:7-8: ISET I 65 ii 1; Ergänzungen nach Ms. Civil): vgl. ebda., Z. 55 lú al-kî̃g-gá ù lú al-me-a ma-[da-àm ár-ni al-AK-e] „Derjenige, der eine Sache in Angrif genommen hat und derjenige, der .... den wird das (ganze) Land rühmen!" (UET 6/2,152:5; Ergänzungen nach Ms. Civil).

11s Vgl. "Enkitalu und Enkiheg̃al“", Z. 178-182 „Meine Mutter ist eine kluge Frau, dic den Haushalt aufgebaut hat. Von den Mägden stehen 10 vor ihr. Mein Vater ist General (und) Richter beim König. Meine Brüder sind ,Gendarme‘ des Königs. Aufseher über 50 (Leute) sind sie. Meine Schwestern stehen wie cin Türflügel im fest gegründeten Frauengemach“: vgl. zu diesen Zcilen Volk, Saeculum 47, 193 mit Anm. 89-91.

116 Aso nicht des Edubbara!

$117 \mathrm{Vgl}$. „Schreiber und Aufseher", Z. 5 [g]i a]-gu ${ }_{4}$-ud-da-gim i-[gu ${ }_{4}$-ud-dè-en] kig̀gá bí-in-sè-ge-en, "Wie ein wiegendes Schilfrohr springe ich umher, in die Arbeit hat er mich gesetzt" (TMHNF 3,38:5 [vgl. C. Wilcke, Kollationen, 35]; SLTNi. 114, side a“ $7+\mathrm{Ni.} 4092$ [ISET II 84] 11; s. zu dicser Zeile B. Alster, Instructions of Suruppak [1974] 93; 131): vgl. weiterhin "Schreiber und Aufseher“. Z. 18 \{u

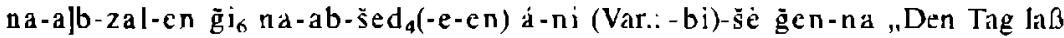
nicht vergehen, die Nacht laß keine Kühle bringen. Geh ihm zur Seite!" (Var.: Zur entsprechenden Arbeit geh!" [SRT 28:12; 3 N-T 917.388 [SLFN 47] 2; Ergänzungen nach Ms. Civil]). 
von früh 118 bis spät die Vorgaben zu erfüllen und niemals zu trödeln ${ }^{119}$, Unter Verleugnung seiner selbst war die Stellung eines Vorgesetzten stets zu respektieren ${ }^{120}$. So sagt der ehemalige Schüler und jetzige Aufseher:

„Die mir (seinerzeit) zuteilgewordenen Worte des Meisters vernachlässigte ich nicht, nichts tat ich von mir selbst aus ${ }^{\text {121. }}$.

Es bedarf wohl kaum der Erklärung, daß eine solche Aussage nichts mehr als die verklärte Retrospektive eines Erwachsenen darstellt ${ }^{122}$, der das Aufbegehren der Jugend nicht anders als mit einer zeitlos stereotypen Formel beantworten wollte oder konnte.

In all diesen Schulstreitgesprächen, die uns einen tiefgehenden Einblick in die Verfahrens- und Denkweise altbabylonischer Erzieher ge-

118 Frübes Aufstehen galt in vielen Kulturen und Phasen der Geschichte als besonders erstrebenswerte Tugend, die es von fruher Kindheit an einzuüben galt. Wenn Xenophon die Bedeutung des frühen Aufstehens im Zusammenhang mit der militïrischen Ausbildung („Denn die Jagd (als Vorbereitung auf die Anfordenungen des Krieges) gewöhnt sie daran. früh aufzustehen und Kālte und Hitze zu ertragen": Xenophon, Kyrupädie - die Erziehung des Kyros [hrsg. und übers. von R. Nickel, 1992] Buch II, 10) betont, so wird das frühe Aufstehen in den Statuten mittelalterlicher (Aus-)Bildungsinstitutionen deshalb geforder1, weil die Aufnahmefähigkeit der Schüler hier am höchsten sei: „Da bei Sonnenaufgang der Lehre und Weisheit Samenkörner geworfen werden sollen und nüchtern das Herz aufnahmefähiger ist, als wenn es von den Reizmitteln des Schlundes angefüllt .... so bestimmen wir: Ein jeder aus den Scholaren des Hauses ,zur Weisheit' [domus sapientic; Var.: sapiencie (Kapitelüberschrift)] soll sich vom Osterfest bis zum Fest des hl. Michacl morgens zur fünften Stunde und vom Fest des hl. Michael bis zum Osterfest zur sechsten Stunde zum Srudium der Wissenschaften erheben ... "Kerer [wie Anm. 68] 53).

119 "Schreiber und Aufseher“, Z. I4 gú-zi-zi ha-la-á-ág-g̃á-kam u-zal-le níğ gig-ga „Frühes Aufstehen ist Bestandteil der Vorschriften. den Tag zu vertrödeln ist ein Tabu“ (SRT 28:8); s. auch „Der Sohn des Tafelhauses“, Z. 17 u na-ab-zalen um-mi-a-ġu $u_{u}$ mu-un-túd-dè-en „Den Tag darf ich nicht vertrödeln, mein Meister wird mich sonst schlugen" (TCL 15,49 r. 17). Die geschilderte Situation trifft aber sicherlich auch für Kinder in einer handwerklichen Ausbildung zu. deren Meister ja ebenfalls $u \mathrm{~m}-\mathrm{mi}$-a genannt wurden.

120 Nur Mutter und Vater, aber auch ein um-mi-a, Meister, der innerhalb eines Lehrverhältnisses Vaterstatt einnehmen konnte, standen in ihrer Autorität über den je. weiligen Vorgesetzten. Sie sind es, die immer wieder mit einem Gott verglichen werden (vgl. Volk, Saeculum 47, 186). In "Schreiber und Aufseher“, Z. 32c (HAV 19:9) spricht der Jungschreiber seinem Aufseher eben diesen Anspruch ab: um-mi-a di.

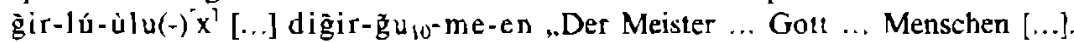
(Und) Du (Aufseher) willst mein Gott sein?" Diese Deutung als Satzfrage ist nach dem Kontext gegeben, entgegen den früheren Ansichten von J. van Dijk, Sagesse (1953) 24; A. Falkenstein, WO 1/3 (1948) $180 \mathrm{f}$.

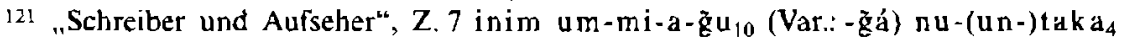
niğ ni-g̃á li-bi-ak (SLTNi. 114, ,side a', Z. 9; TMHNF 3,38:6; Umschrift und Übersetzung berücksichtigen 6 unveröffentlichte Textzeugen [Ms. Civil], da nur TMHNF 3,38:6 [ini]m- 'um-mi-a-gá „die Worte meines Meisters“ bietet).

122 Die alltägliche Realität war, wie die Texte zeigen, oft unerfreulich weit vom Wunschdenken der Erwachsenen entfernt. 
währen, wurde also eine Vielzahl von sozialen Normen ${ }^{123}$, Werten und Inhalten reflektiert und vermittelt. Fand sich ein Kind, ein junger Mensch erst einmal in diesem vielfältigen Geflecht von Wertvorstellungen zurecht und hatte es sich dieser Art von Formung durch die Erwachsenen unterzogen und sie akzeptiert, so konnte man von ihm sagen, er handele vernünftig (úmun-AK) ${ }^{124}$ und sei ein lú- $u_{18}-1 u$, ,(wahrer) Mensch“. Das Menschsein (nam-lú- $\mathrm{u}_{18}-\mathrm{lu}$ ) - im ganz wörtlichen Sinne dem lateinischen humanitas entsprechend 125 - als Erziehungsziel und normative Idee, das befähigt, als Mensch zu handeln; diese gänzlich rationale und säkulare Vorstellung vom Menschsein als Endergebnis einer bewußten Formung ${ }^{126}$ durch Menschen ist es schließlich, die sich Schüler und Absolventen zugute halten oder beim jeweiligen Kontrahenten heftig in Frage stellen. So werfen sie sich immer wieder vor:

.Und du willst ein Mensch sein"??127

oder anders:

„Er ist mehr als du ein Mensch!" ${ }^{228}$

oder:

„Hast du etwa als Mensch gehandelt."

12.3 Vgl. Alster (wic Anm, 61) 8 .

124 Vgl. u.a. „Enkitalu und Enkihegagl“. Z 105 lú úmun nu-mu-e-da-kè-en muda-ab-sá-sá-e-en „Mann, du kannst nicht vernünftig handeln (und) willst mit mir konkurrieren?" (TMHNF 3.42 ii 20); eine noch etwas differenziertere Form des úmun-AK wird König S̆ulgi (Šulgi C. Z. 36 [SRT 14, r. 36]) zugeschrieben: „Ich. Šulgi, der Herrscher über Keng̃ir. der nachweislich vernünftig handelnde" (Š ul-gl lugal ki-en-gi-ra-me-èn úmun-zi-AK-me-èn).

$125 \mathrm{Vgl}$. hierzu schon van Dijk, Sagesse, 23 f; J. Høyrup. nam-lú-ùlu des scribes babyloniens - Un humanisme différent - ma non troppo, in: Michelanea. Humanisme, litteratur og kommunikation $=$ Fs. Michcl Olsen, Sprog og kulturmøde 7 (1994) 73-80. Zur Bestimmung des Terminus, Humanismus' im Zusammenhang der griechischen Paideia vgl. W. Jacger, Paideia. Die Formung des gricchischen Menschen (1989²) $13 \mathrm{f}$

126 „Enkitalu und Enkiheğal, Z.94 gáa-e-gin,-nam al-dim-me-en „So wie ich willst du .geformt" sein?" (TMHNF 3,42 ii 9).

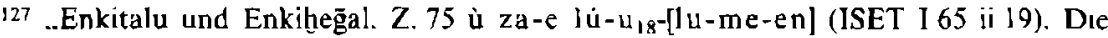
Wendung ist quasi wortgleich zu „Zwei Frauen“(B), Z. 61 (BE 31.28 r. 33) und 96 (BE 31,42:12. Var,: na[m]-lú-u $\mathrm{u}_{18^{\prime}} \mid \mathrm{u}^{\prime}$; CT 58.58:6b). Vgl, zur Deutung dieser Zeilen auch E. I. Gordon, BiOr. 17 (1960) 141، Anm. 15I.

128 „Der Vater und sein mißratener Sohn", Z. 91 diri-zu-šè lú-u $18^{-l u}$ e-ne-àm (Var.:

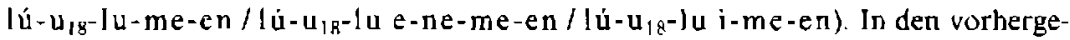
henden Zeilen hatte der Vater seinem Sohn die Kinder anderer Leute vorgehalten, die ihre Eltern, so wie sich das eigentlich und bei entsprechender Erziehung gehört. versorgen. Erst in zweiter Linie (Z. 93) geht es darum, die aufgrund von Ausbildung erworbenen Fertigkeiten des Sohnes als solche in Frage zu stellen.

129 An der von Falkenstein SGL 1,40 partiell zitierten Stelle HAV 19:10 (= ..Schreiber und Aufseher"'. Z. 33) bedeutet na m-lú - ùl u-AK nicht ,zu einem Menschen machen ${ }^{-4}$, 
Auch außerhalb der Schulstreitgespräche wird das Ideal menschenwürdigen Handelns hervorgehoben. So heißt es in einer Hymne an den Gott Enlil:

„In ihr (der Stadt Nippur) respektiert der jüngere Bruder den älteren Bruder, verhält sich (als) Mensch“130.

Daß derlei Idealvorstellungen und -zustände oftmals mehr in Form wohlklingender Reden als durch konkretes Handeln realisiert wurden, galt offenbar als Erfahrungstatsache, die es sehr wohl zu tadeln galt:

„Und du (meinst), Menschsein. das Bestand hat, wäre in irgendeinen ,Mund gefüllt'?"*1.31

Wenn nach dem hier Dargestellten W. Jaeger in seiner auch heute noch referierten „Paideia“ den vorgriechischen Kulturen die Erkenntnis der hohen Bedeutung der Erzichung im weitesten Sinne abspricht und sagt: „Überall wo später in der Geschichte dieser Gedanke (i.e. ,der plastischen Formung von Menschen") wieder auftaucht, ist er ein Erbe der Griechen"132, so ist

sondern "menschlich handeln", ,als menschliches Wesen behandeln": ur-gi $i_{7}-\mathrm{Lur}$ gim igi mu-c-bad-bad nam-lú-ùlu mu-e-ak „Hast du (überhaupl schon) dic Augen wie cin (blind geborener) Welpe geöffnet? Hast du etwa menschlich gehandelt?"Problematisiert wird hier von seiten des Schrejbers das Verhalten des Aufsehers: Letzterer propagiert Maximen der Erziehung, die er nach Ansicht des Schreibers selbst nicht cinlösen kann. Vgl. zu dieser Zeile zuletzt H. Vanstiphout, N.A.B.U. 1996/1 [untcr Angabe der publizierten Texizeugen], der wie folg1 übersetzt: „Once I was but a (blind) puppy; but now my eyes are wide open: now 1 act as a regular human" (Vanstiphouts Interpretation der Zcile als Rede in der I. Person ist nach dem Kontext nicht gegeben. da hier der Schreiber seinem Aufseher in harscher Weise antwortet). Das Bild des Welpen, der nach Ablegen seiner völligen Blindheil zwar schon grobe Umrisse zu sehen vermag, sich aber in seiner Umgebung noch völlig unkontrolliert verhält, spielt auf der Vergleichscbene auch in den Erzichungskonzepten der klassischen Antike eine Rolle: „Indem Xenophon (in seiner Kyropädie) das Wesen und Verhalten des Knaben Kyros mit dem eines Welpen vergleicht, den ja ein unbefangenes, von jeglicher Scheu freies und ungestümes Verhaiten kennzeichnet, bringt er sehr anschaulich zum Ausdruck, daß gerade das gegenteilige Verhalten der Verhaltensnorm entspricht: scheue Zurückhaltung und vornehme Distanz, Respekt, Feingefühl und Takt im Umgang mit anderen ..." (H. Wilms, Techne und Paideia bei Xenophon und Isokrates [1995] 129).

130 „Enlil-sudraše", Z. 32 šeš-gal šeš-bàn-da dugud-dè-dam nam-lú-ùlu kèda m. Die Deutung der Zeile durch Falkenstein (SGL 1,20;39 f.: s. auch D. Reisman, Two Neo-Sum. Hymns [1969] 45) als ,(in ihr) erziehc der älteste Bruder den jüngeren Bruder, macht ihn zu einem Menschen" scheint mir nicht gegeben.

131 "Enkitalu und Enkiheğal“, Z. 116 ù-za-e n[am-lú-ùlu al-gi-na] ka'-b[a a-absi] (ISET 190 ii 8 , ergänzt nach drei unveröffentlichten Textzeugen [Ms. Civil]). TMHNF 3,42 ii 31 bietet an dieser Stelle die Variante ù za-e lú-gi-na ka-bi a$a b-{ }^{\top} j^{1}$, die mir allerdings nicht ganz verständlich ist. Etwa graphisch gegenüber den Parallelen verkürzt?

132 Vgl. Jaeger (wie Anm. 125) 13. 
dieser Ansatz nicht mehr zu rechtfertigen ${ }^{133}$. Zwar ist das für das Alte Griechenland immer wieder herangezogene Bild von der Formung des Menschen ein anderes; denn hier steht die Ausformung des Steinblocks bis hin zur vollendet modellierten Statue als Idee, zu der es vorzudringen gilt, im Vordergrund ${ }^{134}$. In der Welt des alten Babylonien hingegen ist es das der wiederholten Entschlackung und Reinigung, der umfassenden Läuterung des Silbererzes bis hin zu einem perfekt auspolierten Produkt im Wortsinne, wie aus einer Schlüsselaussage des Streitgespräches zwischen einem jungen Schreiber und seinem Aufseher hervorgeht:

„Seit ich klein war, hast du mich großgezogen, hast meinen Lebenswandel beobachtet, hast ihn wie schönes Silber geläutert. (Dabei) kanntest du keıne Grenzen ${ }^{-135}$.

Eine ganz grundlegende Erkenntnis, die sich neben vielem anderem aus den Schulstreitgesprächen des Edubba'a gewinnen läßt, ist also diejenige, daß das alte Babylonien, genauer gesagt, die sumerische Edubba'a-Literatur der altbabylonischen Zeit, eine Metapher für ihre individuelle Vorstellung von Erziehung kennt ${ }^{136}$ : Der Erzieher (des rohstoffarmen Mesopotamien!) als Metallverarbeitender, der das so wertvolle Metall in seiner Materialität und Form verändert und darin nach Vollkommenheit trachtet ${ }^{137}$. Erziehung wird somit, das machen die Schulstreitgespräche deutlich, ganz ausdrücklich als ein Prozeß fortschreitender Wandlung und Vervollkommnung begriffen.

133 Schon H. Brunner. Altägyptische Erziehung (19912) 3. hat sich, bezogen auf die Verhältnisse im Alten Ägypten, sehr kritisch mit der von Jacger vorgetragenen Ansicht auseinandergesetzt. Zwar weicht unter modernen Historikern und Gräzisten eine eindimensionale Sichtweise, wie diejenige Jaegers (und $u$. a. auch von E. A. Havelock), einer zunehmend differenzierteren (W. Burkerl, M. L. West). doch ist der Stellenwert der Thesen Jaegers nach wie vor bedeutsam.

$134 \mathrm{Vgl}$. Jaeger (wie Anm. 125) 12.

$135 \mathrm{Vgl}$ zu dieser Zeile Volk, Saeculum 47. 185, Anm. 45.

136 Vgl. J. Martin/A. Nisschke, Zur Sozialgeschichte der Kindheit (1986) 28, 7u der aus chinesischer Tradition abgeleiteten Leitvorstellung vom Kind als „ungeschliffener Edelstein", den es zu schieifen gilt. Nach G. Linck, ehd. 76, stehen sich in der frühen chinesischen Philosophie zwei Positionen gegenüber. Die daoistische, nach der $p u$, "der unbehauene Holzblock“, als Wesen in seiner naturhaften unberührten Spontaneität zu belassen oder dorthin zurückzuführen sei. Daneben die konfuzianssche, die Erziehung für unabdingbar hielt und dafür die Metapher vom y'u ..Jadestein. der noch geschliffen werden muß" gebraucht.

${ }^{137}$ Ganz eindeutig differenziert hiervon ist die altbabylonisch-akkadische Terminologie, soweil dies aus dem Brief IB I54I (vgl. Anm. 41) zu deduzieren ist: Das zentrale Verbum isı dort wulludum „(cr-)zeugen", "gebären“, ..schaffen”, das über den Umweg der sumerischen Entsprechung (ù-)tu(-d) das Schaffen von Statuen aus einem unbehauenen Steinblock assoziiert, wie dies u. a. wiederholt in den Inschriften des Gudea ausgesagt Ist. Diese Vorsteliung von der Formung des Menschen scheint der oben dargestellten griechischen näher zu stehen. 
Als weiteres und charakteristisches Kennzeichen der in den Schulstreitgesprächen reflektierten Erziehungsvorstellungen läßt sich ihre ausgeprägte Sozialverhaftung ausmachen ${ }^{138}$. Damit ist gemeint, daß die in den Streitgesprächen reflektierten Erziehungsvorstellungen ungebrochen das widerspiegeln, was in der Gesellschaft, die sie hervorgebracht hat, als erstrebens- oder tadelnswert galt ${ }^{139}$.

So hatten die altbabylonischen ummias als Pädagogen und vermutliche Redaktoren der Schulstreitgespräche eine sehr differenzierte Vorstellung von Erziehung ${ }^{140}$. Zwar fand im alten Babylonien, wie nach dem kulturgeschichtlichen Gesamtkontext auch nicht anders zu erwarten, keine Diskussion einer bestimmten Erziehungstheorie, verankert in einem idealisierten Gemeinwesen ${ }^{141}$ oder dem Gesetz, statt, wie dies im Alten Griechenland bei Xenophon, Isokrates oder Platon überliefert ist.

138 Vgl. hierzu auch Høyrup, der bemerkt „Encore une fois, le terme [i. e. na m-lú-àlu] est justifié étymologiquement. awilütum. le mot accadien qui traduit nam-lú-ùlu, signifie aussi l'humanité comprise comme civitas, comme corps social; agir selon awìlūtum veut dire qu'on agit selon les normes de la meilleure sociétéc (wie Anm. 125, 78, Anm. 11)

139 Hierin ist denn auch ein wesentlicher Unterschied gegenüber der Platonschen Erziehungskonzeption, wie erwa in der „politeia“ formuliert, auszumachen: Das Erziehungsziel ist nicht mehr sozial konstituiert, sondern als unwandelbar seiende Idee immer schon gegeben. Dabei gilt es, zu dieser Idee vorzudringen.

Iav Diese könnte, bei aller Problematik des kulturgeschichtlichen Vergleiches, in der täglichen Praxis derjenigen altgriechischen nahegestanden haben, wie sie Platon in seinem großen Alierswerk, den Gesetzen, in ausdrücklicher Abgrenzung gegenüber der reinen Aufzucht und Ausbildung bestimmt: „Nun müssen wir aber das, was wir Bildung und Erziehung nennen, noch erst genauer bestimmen. Denn oft sagen wir eben, um die Erziehung irgend Jemandes von uns zu tadeln oder zu loben, daß der eine Erziehung habe und der andere ohne Erziehung und Bildung sei, obschon der letztere dabei wohl zu den im Kleinhandel und in der Schiffsreederei oder sonstigen in ähnlicher Art in hohem Grade ausgebildeten Leuten gehören kann; und wer sich nun so ausdnïckt, der hält offenbar nicht dies für die Erzehung und Bildung, sondern glaubt, daß nur die von der frühesten Jugend an fortgeführte Erziehung und Heranbildung zu sittlicher Tüchtigkeit diesen Namen (scil, Erziehung/paideia) verdient, welche Lust und Liebe dazu einflößt, ein untadelhafter Bürger zu werden, und das Geschick verlejht. mit Gerechtigkeit zu herrschen und zu gehorchen" (Platon, Gesetze 643e-644a, in der Übersetzung von F. Schleiermacher und F. Susemihl). Man vergleiche hier - als Beispiel - eine von vielen modernen Definitionen von Erziehung. wie sie H. l. Marrou, Histoire de l'éducation dans l'antiquité. ... (1955) 34, gibt. Danach sei Erziehung „une téchnique, par laquelle l'enfant est prèparé et progressivement initié à un mode de vie déterminé, et une éthique, quelque chose de plus qu'une moralè à préceptes: un certain idéal de l'existence, un type idéal d'homme à réaliser".

141 So kritisiert etwa Xenophon in seiner Kyropädie, daß sich die Allgemeinheit zu wenig mit der Erziehung und Ausbildung der griechischen Jugend befasse, hingegen die persische Paideia (als rein fiktive, K.V.) frühzeitig darauf abziele, entsprechende Dispositionen auszubilden (vgl. Wilms [wie Anm. 129] 119). 
Ebensowenig stand die Terminologie als solche im Vordergrund; doch wurde in der Praxis schon bei den Säugelohnverträgen zwischen der rein körperlichen Aufzucht, dem Säugen (šünuqum), und dem „Großziehen ${ }^{\text {" }}$ (tarbirum) unterschieden ${ }^{142}$. Wie sich weiterhin aus den Schulstreitgesprächen ersehen lässt, stand die Erziehung zum Menschen als Ideal, das Menschsein ${ }^{143}$, gebunden an gesellschaftlich bestimmte soziale Normen ausdrücklich neben und über dem Erreichen großer körperlicher oder technischer Geschicklichkeit oder - im weitesten Sinne - handwerksmäßiger Perfektion. Dies zu erkennen und die Fähigkeit zu erwerben, diese Erkenntnis im Sinne der ummias weiterzugeben, war wohl der Prozess, dem sich ein Schüler im Edubba'a zu unterziehen hatte. Erst danach konnte er aus der Sicht der ummias als ein Mensch gelten, der der ihn umgebenden Welt geöffneten Auges begegnen konnte ${ }^{144}$.

Mit dem Zusammenbruch des altbabylonischen Reiches und dem damit verbundenen Niedergang der Edubba'a genannten Institution geht auch die Pflege der Literaturgattung Schulstreitgespräch zugrunde ${ }^{145}$, die außermesopotamisch in der Zeit der Humanisten in besonderer Blüte stand ${ }^{146}$. Einige der in den Schulstreitgesprächen festgehaltenen und ver-

142 Vgl. hier u. a. Codex Ešnunna $\$ 32$ (B ij 13-15) šum-ma LÚ DUMU-šu a-na šu-nu-qíim a-na tar-bi-tim id-di-in-ma ŠE.BA j.BA SIK1.BA MU-3-xAM la id-di-in 10 ma-na tar-biit DUMU-š́ LA்-ma DUMU-šs i-la-a-ar-ru „Wenn ein ,Bürger" sein Kind zum Stillen und Aufziehen gegeben, aber die (vereinbarte) Gerste-, Öl- (und) Wollration über drei Jahre hinweg nicht abgegeben hat, dann wird er 10 Minen (Silber) für die Aufzucht seines Kindes bezahlen und sein Kind zurücknehmen". Nach dieser (und parallelen Aussagen) ist šūnuqum (,stillen“; vgl. tēniqum "Vergütung für das Stillen“: beide Termini begegnen nebeneinander $u$. a. in VS $7,10(/ 11): 4 ; 6)$ als Terminus technicus für die rein körperliche Aufzucht von tarbitum differenziert, das, so es in einem expliziten Gegensatz zu den zuvor genannten Begriffen steht, das Erziehen zu einem altersgemäBen Sozialverhalten bedeutet. Oftmals steht jedoch turbītum in einer Art und Weise für sich, die nahelegt, daB der Terminus an diesen Stellen beide Aspekte. körperliche Aufzucht ebenso wie Erziehung, abdeckt.

143 Der Terminus "Menschsein" sollte allerdings nicht überfordert und aus der Sicht des 20. Jahrhunderts mißdeutet werden. Es handelt sich entsprechend dem kulturgeschichtlichen Gesamtkontext um ein patriarchal strukturiertes Verständnis des Terminus, in den Worten von A. Kessler-Guinan ,... what it means to be human in Mesopotamian terms - more specifically, what it means to be male ..." (Bull of the Canadian Society for Mes. Studies 19 [1990] 11 sub V.).

$144 \mathrm{Vgl}$. hiereu Anm. 5.

145 Auch das oftmals schwer von den Schulstreitgesprächen zu trennende Genre der Schulsatiren (vgl. C. Wilcke, Kindlers Neues Literatur Lexikon [1992] 605), die prominente Nachfolger in den Werken des hellenistischen Mimjambendichters Hero(n)das (Anfang 3. Jhdt. v. Chr.) finden, stirbt mit dem Ende dieser Epoche aus.

146 Der Terminus Renaissance ist an dieser Stelle bewubt vermieden, da sich - zumindestens vorerst - höchstens eine archetypische, aber keinerlei historische Verbindungslinie nach Mesopotamien aufzeigen läßt. 
mittelten Werte werden indes in Mesopotamien weitertradiert und finden sich im I. Jahrtausend, nunmehr verstreut und in ihrer Dialektik modifizert $^{147}$, in Abschnitten der sog. Weisheitsliteratur ${ }^{148}$, der Omenliteratur, hier vor allem in der Serie kataduggû, die F. R. Kraus einst als „,Kanon für die sittliche Haltung des Babyloniers" ${ }^{149}$ bezeichnete, sowie der Beschwörungsliteratur, in Werken wie etwa Šurpu II oder Bit rimki $i^{150}$. In letzterem Werk findet sich ${ }^{151}$, wenn auch nur noch als ferner Anklang an die oben festgemachte Metapher, die Vorstellung vom sündenfreien Menschen als partikelfreies, blank poliertes und strahlendes Objekt aus Edelmetall, das in diesem Zustande Gefallen in den Händen seines Gottes finden kann ${ }^{152}$.

147 Wie beispielsweise in dem starren Protasis/Apodosis-Aufbau der Omenliteratur.

148 Etwa arad mitanguranni, of. W. Röllig, RIA 7 [1987/90] 59, \$4.6.2 b.

149 F. R. Kraus, ZA 43 (1936) 82. Im Zusammenhang mit den Aussagen der Schulstreitgespräche ist hier hervorzuheben, daß auch kuladuggú Bescheidenheit und Zurückhaltung, Selbstbeherrschung, Höflichkeit, Zuverlässigkeit und Hilfsbereitschaft als positive Tugenden charakterisiert. Übcrheblichkeit und Selbstzufriedenheit kommen zu Fall; Fluchen, Schimpfen, Lügen, Verleumden tragen Böses ein.

Isu Vgl. Röllig, RIA 7, 62, \$4.8.2 b

IsI Der Abschnitt des nachfolgend zitierten Textes findet einen terminologischen und sinngemäßen alıbabylonischen Vorläufer $u$. a. in der normalorthographisch geschriehenen Beschwörung YOS 11, 90. Rd, 23-r. 29 (vgl, hierzu zuletzt A. Cavigneaux. ZA 85 [1995] 180 f.).

152 SpTU III Nr. 67 iv 15-22:

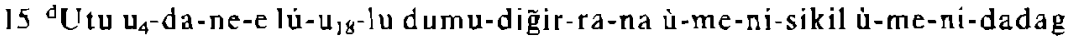
dUTL ina $u_{4}-m i$ an-ni-i a-mi-la mar DIĞIR-šú ul-lil-šú ub-bi-ib-š́u-ma

17 gal-kù-sigir-ga-gim u-me-ni-dadag ki-ma qé-e hu-ra-su nu-um-mir-šú-ma

19 gal-kù-babbar-ra-gim šu u-me-ni-su-ub-su-ub $k i-m a q \dot{e}-e k a j-p i$ šu-kil-šú-mu

$21 \dot{s u-s a t-g a-d i g ̄ i r-r a-n a-s ̌ e ̀ ~ s i l i m-m a-b i ~ h ̧ c ́ c b i-i b ~}$ ana qa-fú dum-qa-fi šá DIG̃̄k-šú šal-mu-us-su pi-qid-su

15 „Utu, hast du an diesem Tage den Menschen, den Sohn seines Gottes, rein, hast du ihn strahlend gemachı (akk.: Šmaš, an diesem Tage, den Menschen. der Sohn seines Gottes, reinige ihn, läutere ihn!).

17 hast du ihn wie ein großes (Gefäß) aus Gold glànzend gemacht, (akk:: Wie ein $q u$-GefäB aus Gold mach' ihn strahlend!)

19 hast du ihn wie ein großes (Gefäß) aus Silber poliert (akk.: Wie ein qù-Gefäß aus Sílber polierc ihn!),

21 dann sollst du ihn zur guten Hand seines Goltes wohlbehalten ... . (akk.: Übergib ihn wohlbehalten der guten Hand seines Gottes!").

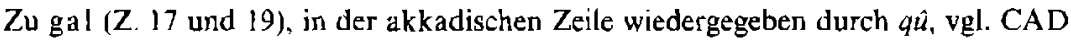
Q 291 sub qû C., bil. sect. mit Parallelen, wo GAt allerdings als Variante zu zabar verstanden wird. Vgl. jedoch W. Sallaberger, Der babyl. Töpfer (1996) 100, sub dug gal, wo uuf gal-Gefäße in der Ur-III-Zeit und in Mari verwiesen wird. Zum Verständnis von gal als Gefä $\beta$, nicht jedoch als Variante zu zaba r „Bronze“, s. auch K. Deller/ K. Watanabe, ZA 70 [1981]211. Anm. 7. 